\title{
Exercise prescription for hospitalized people with chronic obstructive pulmonary disease and comorbidities: a synthesis of systematic reviews
}

This article was published in the following Dove Press journal:

International Journal of COPD

16 May 2012

Number of times this article has been viewed

\author{
W Darlene Reid ${ }^{1,2}$ \\ Cristiane Yamabayashi' \\ Donna Goodridge ${ }^{3}$ \\ Frank Chung ${ }^{4}$ \\ Michael A Hunt ${ }^{1}$ \\ Darcy D Marciniuk ${ }^{5}$ \\ Dina Brooks ${ }^{6}$ \\ Yi-Wen Chen' \\ Alison M Hoens ${ }^{1,7}$ \\ Pat G Camp ${ }^{1,2}$ \\ 'Department of Physical Therapy, \\ University of British Columbia, \\ Vancouver, ${ }^{2}$ Institute of Heart and \\ Lung Health, University of British \\ Columbia, Vancouver, ${ }^{3}$ College of \\ Nursing, University of Saskatchewan, \\ ${ }^{4}$ Physiotherapy, Burnaby Hospital, \\ Fraser Health, British Columbia, \\ ${ }^{5}$ Division of Respirology, Critical \\ Care and Sleep Medicine, University \\ of Saskatchewan, Saskatoon, \\ Saskatchewan, ${ }^{6}$ Department of \\ Physical Therapy, University of \\ Toronto, Toronto, ON, ${ }^{7}$ Providence \\ Health Care, Vancouver, BC, Canada
}

\begin{abstract}
Introduction: The prescription of physical activity for hospitalized patients with acute exacerbation of chronic obstructive pulmonary disease (AECOPD) can be complicated by the presence of comorbidities. The current research aimed to synthesize the relevant literature on the benefits of exercise for people with multimorbidities who experience an AECOPD, and ask: What are the parameters and outcomes of exercise in AECOPD and in conditions that are common comorbidities as reported by systematic reviews (SRs)?
\end{abstract}

Methods: An SR was performed using the Cochrane Collaboration protocol. Nine electronic databases were searched up to July 2011. Articles were included if they (1) described participants with AECOPD, chronic obstructive pulmonary disease (COPD), or one of eleven common comorbidities, (2) were an SR, (3) examined aerobic training (AT), resistance training (RT), balance training (BT), or a combination thereof, (4) included at least one outcome of fitness, and (5) compared exercise training versus control/sham.

Results: This synthesis examined 58 SRs of exercise training in people with AECOPD, COPD, or eleven chronic conditions commonly associated with COPD. Meta-analyses of endurance (aerobic or exercise capacity, 6-minute walk distance - 6MWD) were shown to significantly improve in most conditions (except osteoarthritis, osteoporosis, and depression), whereas strength was shown to improve in five of the 13 conditions searched: COPD, older adults, heart failure, ischemic heart disease, and diabetes. Several studies of different conditions also reported improvements in quality of life, function, and control or prevention outcomes. Meta-analyses also demonstrate that exercise training decreases the risk of mortality in older adults, and those with COPD or ischemic heart disease. The most common types of training were AT and RT. BT and functional training were commonly applied in older adults. The quality of the SRs for most conditions was moderate to excellent $(>65 \%)$ as evaluated by AMSTAR scores.

Conclusion: In summary, this synthesis showed evidence of significant benefits from exercise training in AECOPD, COPD, and conditions that are common comorbidities. A broader approach to exercise and activity prescription in pulmonary rehabilitation may induce therapeutic benefits to ameliorate clinical sequelae associated with AECOPD and comorbidities such as the inclusion of BT and functional training.

Keywords: pulmonary disease, chronic obstructive, comorbidity, exercise, physical fitness

\section{Introduction}

Chronic obstructive pulmonary disease (COPD) is the fifth leading cause of death in the world and the mortality rate is expected to increase more than $30 \%$ during the next 10 years. ${ }^{1}$ Acute exacerbations of COPD (AECOPD) are common and are a key predictor of increased morbidity, health care costs, and mortality. The Global Initiative for Lung Disease (GOLD) ${ }^{1}$ defines a COPD exacerbation as "an event in 
the natural course of the disease characterized by a change in the patient's baseline dyspnea, cough and/or sputum that is beyond normal day-to-day variation, is acute in onset, and may warrant a change in regular medication in a patient with underlying COPD". Most patients with COPD have at least one exacerbation per year; a substantial proportion of patients (17\%) have three or more episodes per year. ${ }^{2,3}$

Many exacerbations of COPD are managed on an outpatient basis, but if the patient is hospitalized, the length of stay (LOS) is prolonged: the median LOS in Canadian hospitals is 13 days, ${ }^{4}$ which translates to an estimated cost of CAD $\$ 9500$ per hospitalization. ${ }^{5}$ Premature discharge resulting in shorter LOS, however, is associated with increased readmission and mortality. ${ }^{6}$

Hospitalization to treat acute illness can have a detrimental effect on muscle performance. ${ }^{7}$ Muscle impairment and functional decline have been well-documented in older hospitalized patients, and have been attributed to the consequences of hospitalization rather than the admitting illness. ${ }^{8-11}$ In a previous study on 1181 hospitalized patients with acute medical illnesses (circulatory, 27\%; respiratory, $20 \%$; gastrointestinal, $15 \%$; cancer, $6 \%$ ), $17 \%$ of patients required assistance with mobility on discharge, despite being able to walk independently prior to admission. ${ }^{12}$ Pitta et al reported that patients who are hospitalized with an acute exacerbation of COPD spend the majority of their time sitting, and do not return to their baseline level of activity even after 1 month post-discharge. ${ }^{13}$

Caring for hospitalized patients with AECOPD can be complicated by the presence of other chronic conditions, which can also influence the severity of the exacerbation and the health outcomes of the patient. Individuals with COPD have a higher risk of multimorbidity ${ }^{14-27}$ (Table 1) than individuals with other chronic illnesses. Despite this, there has been widespread failure to address the complexities inherent in living with multiple chronic illnesses simultaneously, especially in the hospitalized, acutely ill population. Devising exercise plans for hospitalized AECOPD patients living with multimorbidity is complicated by the limitations imposed by the acute exacerbation and by the synergistic effect of the multiple conditions upon the person. While recent reviews have examined evidence regarding the effectiveness of exercise therapy for people with multimorbidity in community settings, ${ }^{28,29}$ none have focused on hospitalized patients with COPD who also experience multi-morbidity. National physical activity guidelines fail to address this issue as well. ${ }^{30,31}$

Systematic reviews (SRs) provide a high level of evidence regarding the efficacy of therapeutic intervention, but the sheer number of available SRs on a given topic can make it difficult for the clinician to distill the important messages. Systematic reviews of systematic reviews have been purported as a way to both evaluate and summarize the key messages on important therapies, and are commonly published in areas of health care. ${ }^{32-36}$ The aim of the current research was to synthesize the relevant literature on the benefits of exercise for people with multimorbidity who are experiencing an AECOPD. Thus we posed the question: What are the parameters and outcomes of exercise in AECOPD and in commonly associated comorbidities as reported by SRs?

\section{Methods}

\section{Search strategy}

A systematic review was performed using the methodology outlined by the Cochrane Collaboration protocol. ${ }^{37}$ Electronic databases were searched up to July 2011 including the Cochrane Controlled Trials Register, Cochrane-Systematic Reviews, MEDLINE, CINAHL (Cumulative Index to Nursing and Allied Health Literature), SPORTDiscus, EMBASE, PEDro (Physiotherapy Evidence Database), PsycINFO, and EBM reviews. Gray literature and reference lists from relevant articles were also reviewed to identify additional articles. Search terms were exemplified by the

Table I Prevalence of chronic diseases in the population and in people with COPD

\begin{tabular}{|c|c|c|}
\hline Chronic disease & Prevalence in population & Prevalence in COPD \\
\hline Heart failure & $<1 \%$ age $50-59 ; \sim 7 \%$ age $80-89^{22}$ & $20 \%{ }^{24}$ \\
\hline Ischemic heart disease & $5 \%$ & Severe and very severe COPD two-fold risk ${ }^{25}$ \\
\hline Peripheral arterial disease & $4.3 \%$ at age $40,14.5 \%$ at age $70^{20}$ & Smokers OR $4.46^{20}$ \\
\hline Hypertension & $15 \% ; \sim 50 \% \geq 50$ years $^{18}$ & I.6-fold risk ${ }^{25}$ \\
\hline Obesity & $67 \%$ over age $30^{14}$ & $54 \%{ }^{26}$ \\
\hline OA & $13 \%$ at age $50 ; \sim 40 \%$ at age $75^{23}$ & \\
\hline Osteoporosis/osteopenia & $15 \%$ over age $50^{21}$ & $60 \%-70 \% 27$ \\
\hline Diabetes mellitus - type 2 & $2.8 \%$ in $2000 ; 6.4 \%$ in 2010 with a $10.2 \%$ in the Western Pacific ${ }^{16}$ & Increased relative risk $1.5-1.8^{25}$ \\
\hline \multirow[t]{2}{*}{ Depression } & $4.7 \%$ persistent depression or anxiety ${ }^{17}$ & Depressive symptoms: $10 \%-80 \%{ }^{25}$ \\
\hline & & Depression that requires treatment: $19 \%-42 \%{ }^{25}$ \\
\hline
\end{tabular}


MEDLINE search strategy (Appendix 1) and were modified accordingly to fit the requirements of the other databases.

\section{Study criteria}

Articles were included if they (1) described participants with AECOPD or COPD, or had one or more of the following conditions that are common comorbidities: anxiety/depression, atherosclerosis, ischemic heart disease, peripheral vascular disease, heart failure, hypertension, diabetes mellitus, human immunodeficiency virus (HIV/AIDS), osteoarthritis (OA), osteoporosis, obesity/overweight, or older adults; (2) were SRs that included at least one randomized controlled trial (RCT); (3) examined an exercise intervention of aerobic training (AT), resistance training (RT), balance training (BT), or a combination thereof; (4) included at least one outcome of fitness; (5) had comparison groups of exercise training versus control/sham (that consisted of usual treatment, no exercise, or attention placebo); (6) were published in English. Articles were excluded if they (1) were published before 2000, or (2) investigated tai chi, yoga, or qi gong due to the multiple forms of these exercises and the difficulty in defining the expected training response to fitness outcomes. If two SRs reported on the same question and the majority of papers included were the same, only the SR with more articles and a more comprehensive review was included. Also, several
SRs did not contain meta-analyses. These were reviewed if they met the criteria above, but data were extracted only if other reviews with meta-analyses were not available on the outcomes of interest.

Two individuals independently screened all titles and abstracts retrieved. Any discrepancies were discussed and resolved. From the selected abstracts, one person screened 362 full text articles to determine if they met the inclusion criteria using a screening form. A second person was consulted to confirm agreement on all articles to be excluded. The flow chart of the search strategy and study selection is summarized in Figure 1.

For each condition, one of the coauthors abstracted data and performed the quality assessment, which was doublechecked by at least one person. All discrepancies were discussed and reconciled. Data abstracted, when available, included (1) condition, age, and gender of participants, (2) the modality (Mo), frequency (Fr), intensity (I), time of session (T), and duration of program (D) of the exercise intervention, (3) descriptors of the control, placebo, or sham group, and (4) outcomes of fitness, disease control or prevention, quality of life, and function.

Data related to functional training was abstracted. Functional training can be defined as mobility exercises that are functionally- or task-based rather than focused on strength

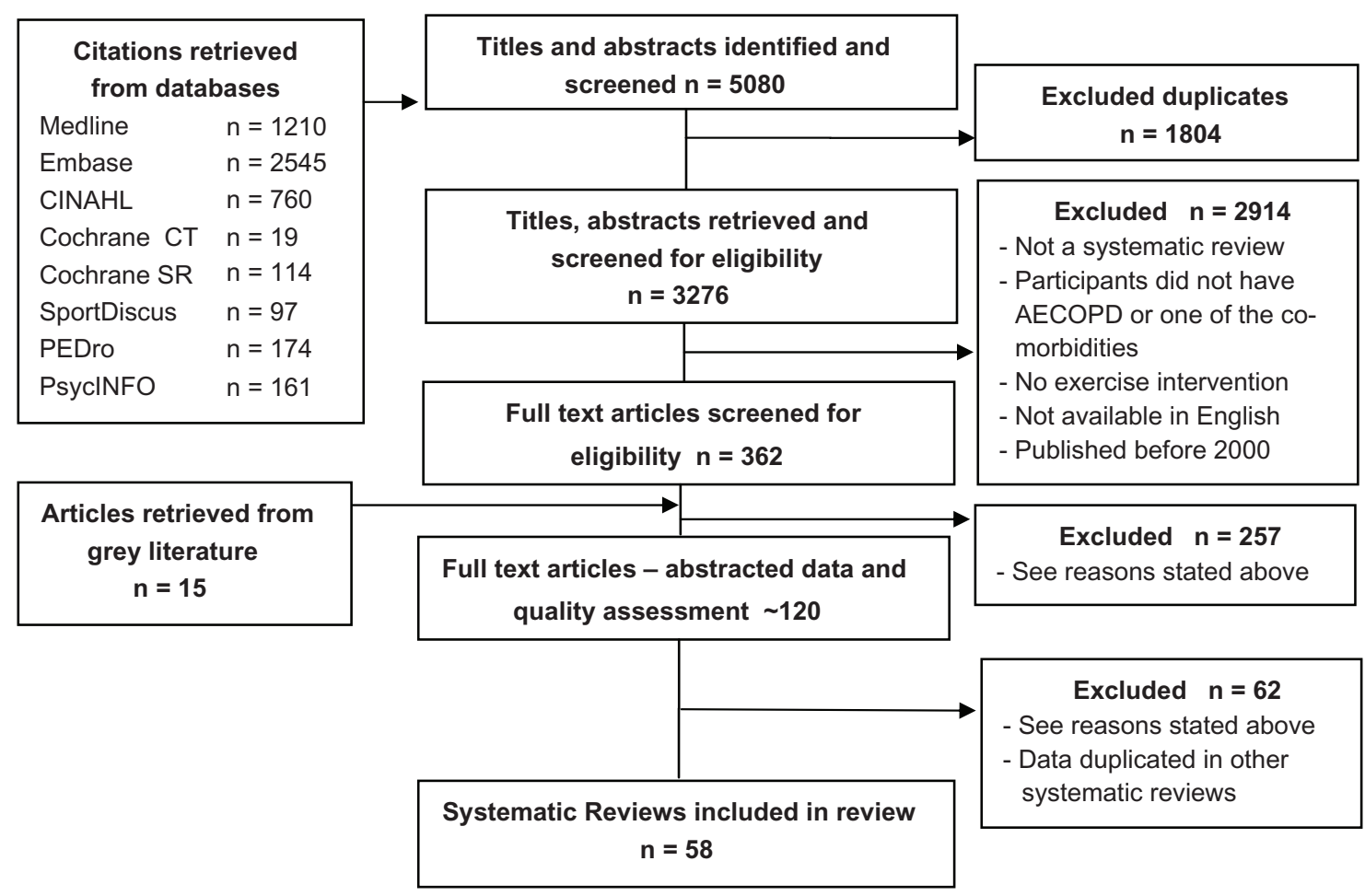

Figure I Flow chart of retrieval, screening and inclusion of systematic reviews. 
training of particular muscle groups or endurance training to improve cardiovascular or aerobic status. Examples are stair climbing, repetitive sit to stand, and transfers. Such training often includes a combination of strength, endurance, and balance.

\section{Quality assessment}

All SRs were assessed using the AMSTAR quality assessment tool. ${ }^{38}$ Scores for each item and totals are reported. Item 11 was scored as "yes" if the conflict of interest was stated for the authors of the SR.

\section{Data synthesis}

Data was synthesized in tables that described the study quality (Table 2), the participants and interventions (Table 3), and outcomes (Table 4). An outcome was assigned to one of two categories based on whether it was measured in several conditions or if it was applied to evaluate a disease specific marker: (1) generic fitness (eg, exercise capacity, strength), quality of life, and functional outcomes (eg, timed up-and-go test, stair climbing), and (2) disease specific fitness, control, and prevention outcomes (eg, dyspnea, mortality, pain, ejection fraction, cholesterol levels).

Only statistically significant outcomes are reported in Table 4. When available, quantitative data for each metaanalysis was described including the number of studies, the number of participants, the standardized effect size or weighted mean difference, risk ratio, odds ratio, and 95\% confidence intervals. If this information was not available, an attempt was made to determine the number of studies that reported a significantly positive change versus the total number of studies that measured the outcome. Nonsignificant findings or trends are not reported.

\section{Results \\ Study selection}

A search of the eight databases yielded 3276 citations and abstracts after duplicates were removed. After independent screening of the citations by two reviewers, 362 full-text articles were selected and reviewed for inclusion. Fifteen additional articles were identified from manual searching of reference lists. The main reasons for excluding articles were that they were not an SR, that participants did not have COPD/AECOPD or one of the other conditions of the search strategy, there was no exercise intervention, the exercise intervention was not clearly described or not differentiated from concurrent treatment, there was no comparison between an exercise group and a control group, the SR reported on similar data but had few articles, the article was not available in English, or the article was published before 2000. No SRs were identified that compared exercise to a control group in people with asthma or bronchiectasis. Fifty-eight SRs met the inclusion criteria.

\section{Quality assessment}

The number of RCTs in any of the SRs that related to our question ranged from 4 to 83 . The average AMSTAR score was 7.5 out of a potential 11 . The most common items not reported were a list of excluded articles (only $26 \%$ of SRs provided this list), an examination of publication bias (ie, funnel plot or Egger regression test, which $45 \%$ reported), a statement regarding conflict of interest (50\% reported), a selection and abstraction of data performed by two reviewers $(65 \%$ reported), and a search of the gray literature (59\% reported). All other items were addressed by $\geq 71 \%$ of the SRs.

\section{Characteristics of participants}

The age of participants ranged between 18 years and the "very elderly". SRs that described people with HIV or depression had the youngest participants. Gender varied from 100\% male to $100 \%$ female. SRs that described older adults had the largest sample sizes.

\section{Characteristics of interventions and outcomes in the different chronic conditions}

Characteristics of the interventions are detailed in Table 3, while outcomes in the different chronic conditions are shown in Table 4.

\section{AECOPD}

One SR investigated the benefits of aerobic and resistance training in people with COPD (AMSTAR of 11). ${ }^{39}$ Aerobic and resistance training of the extremities improved walk distance performance and quality of life measures in addition to decreasing dyspnea, compared to the control group. Exercise also reduced admissions to hospital and mortality as demonstrated by meta-analyses of five and three RCTs, respectively. ${ }^{39}$ The SR included studies that initiated exercise immediately after or up to 6 weeks after treatment initiation for AECOPD as part of pulmonary rehabilitation on an out- or in-patient basis. Frequency of training ranged from three times a week to twice daily. The intensity of aerobic training was not reported.

\section{COPD (stable)}

Six SRs investigated the benefits of AT and RT in people with COPD, and the AMSTAR scores ranged between 5 and $10 .{ }^{40-45}$ 
Table 2 AMSTAR score

\begin{tabular}{|c|c|c|c|c|c|c|c|c|c|c|c|c|c|}
\hline \multirow[t]{2}{*}{ Condition } & \multirow[t]{2}{*}{ Author } & \multicolumn{12}{|c|}{ AMSTAR scores } \\
\hline & & $\bar{I}$ & 2 & 3 & 4 & 5 & 6 & 7 & 8 & 9 & 10 & I I & $\overline{\text { Total }}$ \\
\hline AECOPD & Puhan et $\mathrm{al}^{39}$ & 1 & 1 & 1 & 1 & 1 & 1 & 1 & $\mathrm{I}$ & 1 & 1 & 1 & 11 \\
\hline Chronic obstructive & Lacasse et $\mathrm{al}^{42}$ & I & 1 & 1 & 1 & 1 & I & I & 1 & 1 & 0 & I & 10 \\
\hline \multirow[t]{5}{*}{ pulmonary disease } & O'Shea et al ${ }^{43}$ & $\mathrm{I}$ & $\mathrm{I}$ & I & 0 & 0 & I & I & $\mathrm{I}$ & $\mathrm{I}$ & 0 & I & 8 \\
\hline & Salman et $\mathrm{al}^{44}$ & I & 0 & I & $\mathrm{I}$ & 0 & I & I & I & 1 & 1 & 0 & 8 \\
\hline & Chavannes et $\mathrm{al}^{40}$ & I & 0 & I & I & 0 & I & 0 & 0 & I & 0 & 0 & 5 \\
\hline & Vieira et ll $^{45}$ & I & 0 & I & I & 0 & I & 1 & I & 0 & 0 & 0 & 6 \\
\hline & Janaudis-Ferreira et al $^{4 I}$ & I & 0 & I & I & 0 & I & I & 0 & 0 & 0 & 0 & 5 \\
\hline \multirow[t]{7}{*}{ Older adults } & Liu and Latham ${ }^{50}$ & I & 1 & I & $\mathrm{I}$ & I & I & 1 & I & 1 & 1 & I & 11 \\
\hline & Howe et $\mathrm{al}^{49}$ & 1 & 1 & 1 & 1 & 1 & 1 & 1 & 1 & 1 & 0 & 1 & 10 \\
\hline & Gillespie et $\mathrm{al}^{48}$ & I & I & I & $\mathrm{I}$ & I & I & I & I & I & I & I & $\mathrm{II}$ \\
\hline & Weening-Dijksterhuis et $\mathrm{al}^{52}$ & 1 & 0 & I & I & 0 & I & I & I & 0 & 1 & I & 8 \\
\hline & Forster et $\mathrm{al}^{47}$ & I & I & I & I & 0 & 0 & 0 & 0 & 0 & 0 & I & 5 \\
\hline & Chin et $\mathrm{al}^{46}$ & I & 1 & I & 1 & 0 & $\mathrm{I}$ & I & I & 0 & 0 & I & 8 \\
\hline & Rydwik et $\mathrm{al}^{51}$ & I & 0 & $\mathrm{I}$ & 1 & 0 & I & 1 & $\mathrm{I}$ & 0 & 0 & 0 & 6 \\
\hline \multirow[t]{9}{*}{ Heart failure } & Davies et $\mathrm{al}^{55}$ & $\mathrm{I}$ & 0 & 1 & 0 & 0 & 0 & I & $\mathrm{I}$ & 1 & 1 & 1 & 7 \\
\hline & Hwang et $\mathrm{al}^{54}$ & I & 1 & I & 0 & 0 & I & I & I & I & 1 & 0 & 8 \\
\hline & Hwang and Marwick ${ }^{57}$ & $\mathrm{I}$ & 0 & I & 0 & 0 & I & 0 & 0 & 0 & 0 & 0 & 3 \\
\hline & Chien et $\mathrm{al}^{53}$ & I & I & I & I & 0 & I & I & 0 & 1 & 0 & 0 & 7 \\
\hline & Haykowsky et $\mathrm{al}^{56}$ & I & 1 & 1 & 1 & 0 & I & 1 & I & 0 & 1 & 0 & 8 \\
\hline & van Tol et al ${ }^{58}$ & 1 & 1 & 1 & 0 & 0 & 0 & 1 & I & 1 & 1 & 0 & 7 \\
\hline & Spruit et $a^{61}$ & I & 1 & I & 0 & 0 & I & 1 & I & 0 & 0 & 0 & 6 \\
\hline & Cahalin et $\mathrm{al}^{60}$ & I & 0 & 0 & 0 & 0 & I & 0 & 0 & 0 & 0 & 0 & 2 \\
\hline & Benton $^{59}$ & I & 0 & 0 & 0 & 0 & I & 0 & 0 & 0 & 0 & 0 & 2 \\
\hline \multirow[t]{7}{*}{ Ischemic heart disease } & Haykowsky et al ${ }^{65}$ & I & 1 & I & 0 & 0 & 1 & 1 & I & 1 & 0 & I & 8 \\
\hline & Valkeinen et $a^{68}$ & I & 0 & 0 & 0 & 0 & I & I & 0 & 1 & 1 & 0 & 5 \\
\hline & Cortes et $\mathrm{al}^{64}$ & I & 1 & I & 1 & 0 & I & 1 & I & $\mathrm{I}$ & 0 & I & 9 \\
\hline & Jolliffe et $\mathrm{al}^{66}$ & I & 1 & I & 0 & 1 & I & 1 & I & I & 1 & I & 10 \\
\hline & Clark et $\mathrm{al}^{62}$ & $\mathrm{I}$ & 1 & I & 0 & 0 & $\mathrm{I}$ & 1 & $\mathrm{I}$ & $\mathrm{I}$ & 1 & I & 9 \\
\hline & Cornish et $\mathrm{al}^{63}$ & I & 0 & I & 0 & 0 & I & 0 & 0 & 0 & 0 & 0 & 3 \\
\hline & Oliveira et $\mathrm{al}^{67}$ & I & 0 & I & 0 & 0 & I & 0 & 0 & 0 & 0 & 0 & 3 \\
\hline \multirow[t]{2}{*}{ PVD } & Watson et $\mathrm{al}^{69}$ & I & 1 & I & 0 & I & I & 1 & I & 1 & 0 & I & 9 \\
\hline & Wind and Koelemay ${ }^{70}$ & I & 1 & $\mathrm{I}$ & 0 & 0 & 0 & I & 1 & 1 & 1 & 0 & 7 \\
\hline \multirow[t]{4}{*}{ Hypertension } & Dickinson et $\mathrm{al}^{73}$ & I & 1 & I & 0 & I & 0 & I & I & I & I & 0 & 8 \\
\hline & Cornelissen and Fagard ${ }^{72}$ & I & 1 & 0 & 0 & 0 & 0 & 0 & 0 & 1 & 1 & 0 & 4 \\
\hline & Whelton et $\mathrm{al}^{74}$ & I & I & I & 0 & I & I & 0 & 0 & I & I & I & 8 \\
\hline & Kelley et $\mathrm{al}^{71}$ & 1 & 1 & I & 1 & 0 & 0 & I & 0 & I & 1 & 0 & 7 \\
\hline \multirow[t]{2}{*}{ Obesity } & Shaw et $\mathrm{al}^{76}$ & I & 1 & 1 & 1 & I & I & 1 & 1 & 1 & 1 & I & 11 \\
\hline & Witham and Avenell ${ }^{75}$ & I & 1 & I & 0 & 0 & I & I & 0 & 1 & 1 & I & 8 \\
\hline \multirow[t]{5}{*}{ Osteoarthritis } & Devos-Comby et $\mathrm{al}^{79}$ & I & 0 & I & $\mathrm{I}$ & 0 & $\mathrm{I}$ & I & I & I & 1 & 0 & 8 \\
\hline & Lange et $\mathrm{al}^{80}$ & I & I & I & 0 & 0 & I & I & I & I & 0 & 0 & 7 \\
\hline & Ottawa Panel ${ }^{77}$ & I & I & I & $\mathrm{I}$ & I & $\mathrm{I}$ & I & I & 0 & 0 & 0 & 8 \\
\hline & Brosseau et $\mathrm{al}^{78}$ & I & I & I & I & 0 & I & I & I & 0 & 0 & 0 & 7 \\
\hline & Pelland et $a^{81}$ & I & I & I & I & I & I & I & I & I & 0 & 0 & 9 \\
\hline Osteoporosis & Li et $\mathrm{al}^{83}$ & 1 & 0 & 1 & 0 & 0 & 0 & 1 & 1 & 1 & 0 & 1 & 6 \\
\hline & de Kam et $\mathrm{al}^{82}$ & 1 & 0 & 1 & 1 & 0 & 1 & 1 & 1 & 0 & 0 & 1 & 7 \\
\hline Diabetes mellitus & Chudyk and Petrella ${ }^{85}$ & I & 0 & I & I & 0 & I & 0 & 0 & 1 & 0 & I & 6 \\
\hline & Umpierre et $\mathrm{al}^{90}$ & I & I & I & $\mathrm{I}$ & 0 & I & I & $\mathrm{I}$ & I & I & I & 10 \\
\hline & Irvine and Taylor ${ }^{86}$ & 1 & 1 & I & 0 & 0 & I & I & I & I & 0 & 0 & 7 \\
\hline & Thomas et $\mathrm{al}^{89}$ & I & 1 & 1 & 1 & I & I & I & I & I & 1 & I & $\mathrm{II}$ \\
\hline & Kelley and Kelley ${ }^{87}$ & I & 1 & I & 1 & 0 & I & I & I & I & 1 & 0 & 9 \\
\hline & Snowling and Hopkins ${ }^{88}$ & I & 0 & I & 0 & 0 & I & I & $\mathrm{I}$ & I & 0 & 0 & 6 \\
\hline & Boulé et al ${ }^{84}$ & I & 0 & I & 1 & 0 & I & 1 & 0 & 1 & 0 & 0 & 6 \\
\hline HIV & O'Brien et $\mathrm{a}^{91}$ & I & I & I & 1 & 1 & I & 1 & I & 1 & 0 & 1 & 10 \\
\hline Depression & Krogh et $\mathrm{al}^{93}$ & I & I & I & $\mathrm{I}$ & 0 & I & I & I & $\mathrm{I}$ & I & I & 10 \\
\hline & Herring et $\mathrm{al}^{94}$ & I & 0 & I & I & 0 & 0 & I & I & I & I & I & 8 \\
\hline & Mead et $\mathrm{al}^{96}$ & I & I & I & I & I & I & I & I & I & i & I & 11 \\
\hline & Rethorst et al $^{95}$ & 1 & 1 & I & 1 & 0 & I & 1 & 0 & 1 & 0 & 1 & 8 \\
\hline & Lawlor and Hopker ${ }^{92}$ & I & I & 1 & 1 & 0 & I & I & I & I & 0 & I & 9 \\
\hline Tallies for 58 reports & & 58 & 38 & 54 & 34 & 15 & 49 & 48 & 41 & 42 & 26 & 29 & 434 \\
\hline Percentage of total (58 & orts) & 100 & 66 & 93 & 59 & 26 & 84 & 83 & 71 & 72 & 45 & 50 & \\
\hline
\end{tabular}


Table 3 Characteristics of participants and interventions

\begin{tabular}{|c|c|c|c|c|}
\hline Author & RCTs & n (\% $M$ or $F)$ & Age (years) & Characteristics of exercise intervention \\
\hline \multicolumn{5}{|l|}{ AECOPD } \\
\hline Puhan et a ${ }^{39}$ & 9 & $427(64 \% \mathrm{M})$ & Range $62-70$ & $\begin{array}{l}\text { Mo: AT and RT; Fr: Twice daily }-3 \times / \text { week; } \\
\text { T: NR except one study stated } 2 \text { hours/session; } \\
\text { D: } 10 \text { days }-6 \text { months }\end{array}$ \\
\hline \multicolumn{5}{|l|}{ COPD } \\
\hline Lacasse et $\mathrm{al}^{42}$ & 31 & 1322 & NR & $\begin{array}{l}\text { Mo: AT and RT, U/E, L/E, and/or respiratory } \\
\text { muscle training; Fr and I: NR; D: 4-52 weeks }\end{array}$ \\
\hline O'Shea et al ${ }^{43}$ & 9 of 18 & 296 of $679(70 \% \mathrm{M})$ & Range 48.5-72 & $\begin{array}{l}\text { Mo: RT using free weights, machine, Theraband; } \\
\text { Fr: } 2 \times / \text { week-daily; I: variable; T: 5-12 reps, } \\
\text { 2-4 sets; D: 6-12 weeks }\end{array}$ \\
\hline Salman et $\mathrm{a}^{44}$ & 20 & 999 & $59-73$ & $\begin{array}{l}\text { Mo: AT, RT: U/E, L/E, and/or respiratory muscle } \\
\text { training; Fr: } \geq 3 \times / \text { week; D: } 6 \text { weeks-1 } 2 \text { months; } \\
\text { other details NR }\end{array}$ \\
\hline Chavannes et $\mathrm{a}^{40}$ & 4 of 5 & $210(\sim 55 \% \mathrm{M})$ & $49-63$ & $\begin{array}{l}\text { Mo: AT + RT (four studies), AT (one study); } \\
\text { Fr: } 2-7 \times / \text { week; D: } 8 \text { weeks-1 } 8 \text { months; } \\
\text { other details NR }\end{array}$ \\
\hline Vieira et $\mathrm{a}^{45}$ & 8 of 12 & 370 of 728 & Range 38-78 & $\begin{array}{l}\text { Mo: Home-based AT and RT; Fr: } 2 \times / \text { week }- \text { twice } \\
\text { daily; I: } 90 \% \text { of velocity of } 6 \mathrm{MWD}, \geq 70 \% \\
\text { of maximal speed of SWT, cycle ergometer: } \\
30 \mathrm{~W} \text {, cycling: } 70 \% \text { of work rate; } \\
\text { T: } 30-60 \text { minutes; D: } 4-52 \text { weeks }\end{array}$ \\
\hline $\begin{array}{l}\text { Janaudis-Ferreira } \\
\text { et al }\left.\right|^{41}\end{array}$ & 5 & 157 & NR & $\begin{array}{l}\text { Mo: Arm AT and RT; Fr: } 3 \times / \text { week }-2 \times / \text { day; } \\
\text { I: NR; T: } 4-10 \text { reps I-3 sets; D: } 6-8 \text { weeks }\end{array}$ \\
\hline \multicolumn{5}{|l|}{ Older adults } \\
\hline Liu and Latham ${ }^{50}$ & 83 of $|2|$ & 3059 of 6700 & $>60$ & $\begin{array}{l}\text { Mo: RT in gym or against Theraband; } \\
\text { Fr: } 2-3 \times / \text { week, except one was daily; I: ranged } \\
\text { from low to high intensity; D: most } 8-12 \text { weeks } \\
\text { but ranged from 2-104 weeks }\end{array}$ \\
\hline Howe et al ${ }^{49}$ & 34 & $2883(<50 \% \mathrm{M})$ & $60-75$ years & $\begin{array}{l}\text { Mo: RT, gait, balance, co-ordination, and functional } \\
\text { tasks, tai chi, qi gong, dance, yoga; other details NR }\end{array}$ \\
\hline Gillespie et $\mathrm{a}^{48}$ & I4 of III & $55303(<50 \% \mathrm{M})$ & $>60$ & $\begin{array}{l}\text { Mo: AT, RT, gait, balance and functional training, } \\
\text { flexibility training, tai chi, and square stepping; } \\
\text { general physical activity (walking groups); } \\
\text { other details NR }\end{array}$ \\
\hline $\begin{array}{l}\text { Weening- } \\
\text { Dijksterhuis et } \mathrm{a}^{52}\end{array}$ & 27 & 6459 & $>70$ & $\begin{array}{l}\text { Mo: RT (strength, resistance), ROM, balance, } \\
\text { functional, gait, tai chi, flexibility; other details NR }\end{array}$ \\
\hline Forster et al ${ }^{47}$ & 37 of 49 & $\begin{array}{l}3611 \\
(33 \% M)\end{array}$ & Mean 82 & $\begin{array}{l}\text { Mo: RT, AT (walking) and general daily living skills eg, } \\
\text { eating, dressing, and climbing stairs, flexibility, balance; } \\
\text { Fr: mean of } 3.5 \times / \text { week } \mathrm{T}: \leq 30 \text { minutes/week; } \\
\text { D: } 4 \text { weeks }-2 \text { years }\end{array}$ \\
\hline Chin et $\mathrm{a}^{46}$ & 20 & 2515 (majority F) & $77-88$ & $\begin{array}{l}\text { Mo: AT, RT, flexibility, balance, tai chi, and/or } \\
\text { multi-component training; Fr: usually } 3 \times / \text { week; } \\
\text { D: range } 10-28 \text { weeks }\end{array}$ \\
\hline Rydwik et $\mathrm{al}^{51}$ & 16 & 1269 & $>70$ & $\begin{array}{l}\text { Mo: RT, gait, and ROM training; Fr: usually } 2-3 \times / \text { week } \\
\text { T: variable; } \mathbf{D}: 9 \text { weeks }-2 \text { years }\end{array}$ \\
\hline \multicolumn{5}{|l|}{ Heart failure } \\
\hline Davies et $\mathrm{a}^{55}$ & 19 & 3647 (majority M) & $5 I-72$ & $\begin{array}{l}\text { Mo: AT or AT + RT; } \mathbf{F r}: 2-7 \times / \text { week; I: } 40 \% \max \\
\text { HR or } 85 \% \mathrm{VO}_{2} \max ; \text { T: I5-1 } 20 \text { minutes; } \\
\text { D: } 24 \text { weeks-3 years }\end{array}$ \\
\hline Hwang et al ${ }^{54}$ & 4 of 8 & $103(60 \% \mathrm{M})$ & $62-77$ & $\begin{array}{l}\text { Mo: RT for U/E and L/E; Fr: 2-3×/week; } \\
\text { I: } 60 \%-80 \% \text { IRM; T: I2-60 minutes; D: 8-22 weeks }\end{array}$ \\
\hline $\begin{array}{l}\text { Hwang and } \\
\text { Marwick }^{57}\end{array}$ & 19 & $1069(85 \%$ M) & $\begin{array}{l}59.05 \\
(95 \% \mathrm{Cl} 55-63)\end{array}$ & $\begin{array}{l}\text { Mo: AT or AT + RT: cycle ergometer, walking, } \\
\text { calisthenics, ball games, strength training; Fr: NR; } \\
\text { I: AT: }-10 \% \text { of AT, } 60 \%-80 \% \text { HRmax, } 70 \% \mathrm{VO}_{2} \max \\
\text { RT: } 80 \% \text { IRM, } 70 \% \text { HRmax; } \\
\text { T: } 40-400 \text { minutes/week; D: } 8-52 \text { weeks }\end{array}$ \\
\hline
\end{tabular}


Table 3 (Continued)

\begin{tabular}{|c|c|c|c|c|}
\hline Author & RCTs & $n(\%)$ or $F)$ & Age (years) & Characteristics of exercise intervention \\
\hline Chien et $\mathrm{a}^{53}$ & 10 & $648(79 \% \mathrm{M})$ & $52-81$ & $\begin{array}{l}\text { Mo: Home based AT (walking program, and/or cycle } \\
\text { ergometer) and RT (added in 3/I0 studies); } \\
\text { Fr: } 2-5 \times / \text { week; I: } 40 \%-70 \% \text { HRmax, or } 70 \% \text { HR } \\
\text { at } \mathrm{VO}_{2} \text { max; T: } 20-60 \text { minutes; D: 6-52 weeks. } \\
\text { All home programs }\end{array}$ \\
\hline Haykowsky et a ${ }^{56}$ & 9 of 14 & $\begin{array}{l}538 \text { of } 812 \\
(83 \% \mathrm{M})\end{array}$ & $52-61$ & $\begin{array}{l}\text { Mo: AT: cycle ergometer, rowing, swimming, walking, } \\
\text { arm ergometer, treadmill, interval training; Fr: } 2-7 \\
\text { days/week; I: } 50 \%-80 \% \mathrm{VO}_{2} \text { peak, } 60 \%-85 \% \text { HRpeak, } \\
\text { or } 50 \%-70 \% \text { max work load; T: } 20-60 \text { minutes; D: } \\
2-14 \text { months }\end{array}$ \\
\hline van Tol et al ${ }^{58}$ & 35 & I 486 (76\% M) & $\begin{array}{l}\text { Mean } 60.6 \\
(S D 7.5)\end{array}$ & $\begin{array}{l}\text { Mo: AT + RT; Fr: range I-7 } 7 \times / \text { week, } \\
\text { mean } 3.7 \times / \text { week; I: } 50 \%-80 \% \mathrm{VO}_{2} \text { peak, } 60 \%-80 \% \\
\text { HRmax, } 60 \%-80 \% \text { HRR; T: I5-96 minutes, } \\
50 \text { minutes (mean); D: } 3-26 \text { weeks, I } 3.0 \text { weeks (mean) }\end{array}$ \\
\hline Spruit et $\mathrm{a}^{61}$ & 4 of 10 & II4 (72\% M) & Mean 57-77 & $\begin{array}{l}\text { Mo: } \mathrm{RT} \text { alone } \mathbf{F r} \text { : } 2-3 \times / \text { week; I: } 40 \%-80 \% \text { I RM; } \\
\text { T: I5-60 reps in I-3 sets; D: } 8-20 \text { weeks; progression } \\
\text { described in some reports }\end{array}$ \\
\hline Cahalin et $\mathrm{al}^{60}$ & 11 of 22 & $\begin{array}{l}387 \text { of } 633 \\
(70 \% \mathrm{M})\end{array}$ & $30-80$ & $\begin{array}{l}\text { Mo: RT: variety of strength exercises either alone or } \\
\text { with short or long bursts of AT; } \mathbf{F r}: 2-5 \times / \text { week; } \\
\text { I: RT: } 20 \%-80 \% \text { IRM; AT: up to } 80 \% \mathrm{VO}_{2} \text { peak, } \\
\text { or } 70 \% \text { HRpeak, or } 50 \% \text { peak workload; } \\
\text { T: } 5-60 \text { minutes; D: } 4-26 \text { weeks }\end{array}$ \\
\hline Benton $^{59}$ & 4 of 16 & $\begin{array}{l}\text { II of } 379 \\
(80 \% \mathrm{M})\end{array}$ & Mean 30-76 & $\begin{array}{l}\text { Mo: RT: chair stands, heel lifts, weights, pulleys, } \\
\text { hydraulic fitness; Fr: } 2-3 \times / \text { week, I: } 60 \% \text { I RM; AT: } \\
\text { cycling, arm ergometer, stair climbing, walking; } \\
\text { T: } 2-60 \text { minutes; D: } 2-5 \text { month study duration; reps } \\
\text { and progression: variable }\end{array}$ \\
\hline \multicolumn{5}{|c|}{ Ischemic heart disease } \\
\hline Haykowsky et al ${ }^{65}$ & 12 & 647 & 55 & $\begin{array}{l}\text { Mo: AT: Cycle, walk, jogging, calisthenics; } \\
\text { Fr: } 3-7 \times / \text { week; I: } 60 \%-80 \% \mathrm{VO}_{2} \text { max or peak HR; } \\
\text { T: } 30-180 \text { minutes; D: I-6 months }\end{array}$ \\
\hline Valkeinen et al ${ }^{68}$ & 18 & 922 (majority M) & $59.9 \pm 4.9$ & $\begin{array}{l}\text { Mo: AT: walking, jogging, cycling, and arm cranking; } \\
\text { Fr: } 3 . I \pm 0.4 \times / \text { week; I: } 70 \%-80 \% \text { HRmax, HRR, } \\
\text { HR of anaerobic threshold or } 25 \%-70 \% \mathrm{VO}_{2} \text { max; } \\
\text { T: } 20-60 \text { minutes; D: I4.2 } \pm \text { I3.5 weeks } \\
\text { Mo: RT: Fr: } 3.5 \pm 0.7 \times / \text { week; I: } 50 \%-80 \% \text { I RM or } \\
\text { 40\%-60\% MVC; T: } 20-60 \text { minutes; D: } 22.0 \pm \text { I I.I weeks }\end{array}$ \\
\hline Cortes et $\mathrm{al}^{64}$ & II of 14 & $3 \mid 48(\geq 70 \% M)$ & $55-65$ & $\begin{array}{l}\text { Mo: Early mobilization: sitting on bed/chair, walking, } \\
\text { moving legs, climbing stairs, any movement out } \\
\text { of bed, dangling legs, progressive activity while } \\
\text { in hospital; settings descriptions: CCU, } \\
\text { hospital wards, in-hospital }\end{array}$ \\
\hline Jolliffe et $\mathrm{a}^{166}$ & 32 & 8440 (majority M) & 55 & $\begin{array}{l}\text { Mo: AT: walking, running, cycling, skipping, swimming, } \\
\text { stairs, and rowing; Fr: I-5×/week; I: } 70 \%-85 \% \\
\text { HRmax or predicted HRmax, other } 75 \% \text { max work } \\
\text { capacity; T: } 20-60 \text { minutes; D: } 4 \text { weeks to } 30 \text { months } \\
\text { Mo: RT: strengthening exercises for U/E and L/E; } \\
\text { Fr: } 3 \times / \text { week; I: Target HR } 85 \% \text { HRmax; } \\
\text { T: } 60 \text { minutes; D: } 12 \text { weeks }\end{array}$ \\
\hline Clark et al ${ }^{62}$ & 41 of 63 & $\begin{array}{l}8460 \text { of } 21295 \\
\text { (majority M) }\end{array}$ & Mean 49-7I & AT, RT: no details on intervention \\
\hline Cornish et a ${ }^{63}$ & 2 of 7 & 120 of 213 & $57 \pm|4-7| .5 \pm 7.8$ & $\begin{array}{l}\text { Mo: AT: interval training: aerobic dance movements, } \\
\text { upper/lower body exercises, cycle ergometer, or } \\
\text { treadmill walking/running; Fr: } 2-3 \times / \text { week; I: } 3 \text { intervals } \\
\text { of } 5-10 \text { minutes at RPE I5-18 in one study and } 3 \\
\text { minutes at } 60 \%-70 \% \text { HRmax }+4 \text { minutes at } 90 \%-95 \% \\
\text { HRmax in another; T: } 50-60 \text { minutes; D: } 16-26 \text { weeks }\end{array}$ \\
\hline
\end{tabular}

(Continued) 
Table 3 (Continued)

\begin{tabular}{|c|c|c|c|c|}
\hline Author & RCTs & n (\% M or F) & Age (years) & Characteristics of exercise intervention \\
\hline Oliveira et $\mathrm{al}^{67}$ & 4 of II & 126 & most $<60$ & $\begin{array}{l}\text { Mo: RT: free weights in one study, NR in others; } \\
\text { I: } 40 \%-60 \% \text { MVC or } 50 \%-80 \% \text { I RM; D: } 10 \text { weeks } \\
\text { to } 6 \text { months; Other details NR }\end{array}$ \\
\hline \multicolumn{5}{|l|}{ PVD } \\
\hline Watson et $\mathrm{al}^{69}$ & 16 of 22 & $\begin{array}{l}783 \text { of } 1200 \\
(>50 \% \mathrm{M})\end{array}$ & $45-89$ & $\begin{array}{l}\text { Mo: AT, RT: polestriding, cycling, and U/E and L/E } \\
\text { exercises. Fr: } 2-7 \times / \text { week; T: } 30-120 \text { minutes; } \\
\text { D: } 3-12 \text { months }\end{array}$ \\
\hline $\begin{array}{l}\text { Wind and } \\
\text { Koelemay }{ }^{70}\end{array}$ & 10 of 15 & $\begin{array}{l}625 \text { of } 76 \mathrm{I} \\
(>50 \% \mathrm{M})\end{array}$ & $60-76$ & $\begin{array}{l}\text { Mo: AT: crank exercise, walking, others not } \\
\text { detailed. Fr: 2-3x/week; T: } 30-60 \text { minutes; } \\
\text { D: } 12-26 \text { week except one study was } 104 \text { weeks }\end{array}$ \\
\hline \multicolumn{5}{|l|}{ Hypertension } \\
\hline Dickinson et $\mathrm{al}^{73}$ & 21 of 105 & $\begin{array}{l}\text { I5I8 of } 6805 \\
(\sim 57 \% \mathrm{M})\end{array}$ & $52(30-67)$ & $\begin{array}{l}\text { Mo: AT: brisk walking, jogging, cycling; Fr: } 3-5 \times / \text { week; } \\
\text { T: } 30-60 \text { minutes; D: } 8-52 \text { week (median } 12 \text { ). } \\
2 \text { trials received RT, but details NR; I trial offered } \\
\text { advice to participants }\end{array}$ \\
\hline $\begin{array}{l}\text { Cornelissen } \\
\text { and Fagard }{ }^{22}\end{array}$ & 28 of 72 & $\begin{array}{l}492 \text { in exercise } \\
\text { group }\end{array}$ & $52.7 \pm 11.8$ & $\begin{array}{l}\text { Mo: AT: walking, jogging, running, cycling; } \\
\text { Fr: } 2-7 \times / \text { week (median 3); I: } 30 \%-87 \% \text { HRR } \\
\text { (median 65); T: } 25-60 \text { minutes (median 40); } \\
\text { D: 4-52 weeks (median 12) }\end{array}$ \\
\hline Whelton et $\mathrm{al}^{74}$ & 15 of 54 & 872 (majority M) & $\begin{array}{l}\text { Mean 40-69 } \\
\text { Range 18-86 }\end{array}$ & $\begin{array}{l}\text { Mo: AT: biking, walking, jogging; Fr: I-7 } \times / \text { week; } \\
\text { I: } 40 \%-70 \% \mathrm{VO}_{2} \max , 60 \%-85 \% \mathrm{HRmax}, 40 \%-70 \% \\
\text { Wmax; T: } 20-60 \text { minutes; D: 3-26 weeks }\end{array}$ \\
\hline Kelley et $\mathrm{al}^{71}$ & 47 & $\begin{array}{l}2543 \\
>50 \% M\end{array}$ & 48 & $\begin{array}{l}\text { Mo: AT: walking, jogging, cycling, aerobic dance, } \\
\text { and swimming; Fr: I-5×/week; I: } 45 \%-86 \% \mathrm{VO}_{2} \max \\
\text { T: I5-60 minutes; D: } 360-9360 \text { minutes }\end{array}$ \\
\hline \multicolumn{5}{|l|}{ Obesity } \\
\hline Shaw et $\mathrm{a}^{76}$ & 4 of 41 & $\begin{array}{l}440 \text { of } 3476 \\
\text { (majority M) }\end{array}$ & $30-64$ & $\begin{array}{l}\text { Mo: AT: walking/jogging, circuit training; } \\
\text { Fr: 2-3x/week; I: } 60 \%-80 \% \text { HRmax; } \\
\text { T: } 30-60 \text { minutes; D: } 26-52 \text { weeks }\end{array}$ \\
\hline $\begin{array}{l}\text { Witham } \\
\text { and Avenell }{ }^{75}\end{array}$ & 2 of 9 & 173 (I00\% F) & 60 & $\begin{array}{l}\text { Mo: supervised AT, no details; Fr: } 3 \times / \text { week; } \\
\text { I: aiming for } 70 \% \mathrm{VO}_{2} \text { max; T: NR; D: } 3 \text { months; } \\
\text { Transition to home: exercise } 4 \times / \text { week plus } \\
\text { weekly supervised exercise }\end{array}$ \\
\hline \multicolumn{5}{|r|}{ 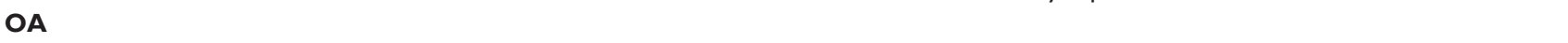 } \\
\hline $\begin{array}{l}\text { Devos-Comby } \\
\text { et } \mathrm{al}^{79}\end{array}$ & 16 & 2154 & $\begin{array}{l}\text { Mean 65.8, } \\
\text { range 29-89 }\end{array}$ & $\begin{array}{l}\text { Mo: AT + RT: knee muscles strength training, } \\
\text { low/medium intensity exercise, and light physical } \\
\text { activity (walking, aerobic exercise, balance or flexibility } \\
\text { exercises); Fr: } 2-5 \times / \text { week (sometimes } 2 \times / \text { day); } \\
\text { I: AT: } 50 \%-85 \% \text { HRR, RT: } 6 \text { set } \times 5 \text { max contraction } \\
\text { (others NR); T: } 20 \text { minutes }-1.5 \text { hour; } \\
\text { D: } 4-12 \text { weeks (different follow-up periods) }\end{array}$ \\
\hline Lange et $\mathrm{a}^{80}$ & 18 & 2723 & Range 55-74 & $\begin{array}{l}\text { Mo: RT: dynamic or isotonic training mainly targeting } \\
\text { the quadriceps using resistance machines, free weights, } \\
\text { or Theraband; Fr: } 2-7 \times / \text { week; I: light to maximal; } \\
\text { T: I0-60 minutes, } 5-12 \text { reps, I-10 sets (most } 3 \text { sets); } \\
\text { D: I-30 months }\end{array}$ \\
\hline Ottawa Panel ${ }^{77}$ & 26 & 2486 & $>18$ & $\begin{array}{l}\text { Mo: AT: walking or cycle ergometer; RT: strengthening } \\
\text { (eg, isometric, isotonic, isokinetic, eccentric, concentric, } \\
\text { aerobic), general physical activity, combination of } \\
\text { exercises; Fr: IX/week-10×/day (depends on phase); } \\
\text { I: AT: } 50 \%-70 \% \text { HRR when described, RT: variable. } \\
\text { T: } 5 \text { minutes-I.5 hours; D: } 4 \text { weeks-18 months }\end{array}$ \\
\hline Brosseau et al ${ }^{78}$ & 9 of 12 & 1363 & $57-69.4$ & $\begin{array}{l}\text { Mo: AT: walking, stationary bike; Fr: I-4 } \times / \text { week; } \\
\text { I: } 50 \%-85 \% \text { HRR or } 60 \%-80 \% \text { HRmax. T: } 30-90 \\
\text { minutes when reported; D: } 5-12 \text { weeks. Some changed } \\
\text { to home-based program up to } 15 \text { months. Some } \\
\text { studies included strengthening exercises }\end{array}$ \\
\hline
\end{tabular}


Table 3 (Continued)

\begin{tabular}{|c|c|c|c|c|}
\hline Author & RCTs & $n(\% M$ or $F)$ & Age (years) & Characteristics of exercise intervention \\
\hline Pelland et a $\left.\right|^{81}$ & $\begin{array}{l}\text { I7 } \\
\text { (plus } 4 \text { CCTs) }\end{array}$ & 2325 & Range $55.0-74.6$ & $\begin{array}{l}\text { Mo: ROM exercises, low intensity walking on treadmill, } \\
\text { RT: quadriceps, hamstrings, and hip abduction } \\
\text { strengthening, stationary bike; Fr: most } 2-3 \times / \text { week, } \\
\text { range: IX/week-2×/day; I: variable; T: I5-40 minutes, } \\
\text { 3-9 exercises, } 6-20 \text { reps, I-4 sets; } \\
\text { D: } 4 \text { week-I5 months }\end{array}$ \\
\hline \multicolumn{5}{|l|}{ Osteoporosis } \\
\hline Li et $\mathrm{al}^{83}$ & $\begin{array}{l}5 \text { groups in } \\
4 \text { papers }\end{array}$ & $256(100 \% \mathrm{~F})$ & NR & $\begin{array}{l}\text { Mo: RT or combined stretch/strength/balance } \\
\text { programs: strengthening of extremities and trunk } \\
\text { (I study), "agility" training (I study), combined exercise } \\
\text { (stretching, strengthening, balance, posture) ( } 2 \text { studies), } \\
\text { trunk flexor/extensor strengthening (I study); } \\
\text { Fr: } 3 \text { studies } 2 \text { days/week, I study } 7 \text { days/week; I: NR; } \\
\text { T: } 40-60 \text { minutes; D: 10-25 weeks }\end{array}$ \\
\hline De Kam et $\mathrm{al}^{82}$ & 28 & $\begin{array}{l}\text { I707, some reports } \\
\text { used the same } \\
\text { subjects }\end{array}$ & Mean 57-82 & $\begin{array}{l}\text { Mo: fast walking; aerobic exercise; weight-bearing } \\
\text { aerobic exercise; strengthening exercises; balance } \\
\text { exercises; posture and gait exercises; trunk extensor } \\
\text { exercises; heel drops with impact; heel drops with } \\
\text { no impact; whole body vibration; jumping; agility } \\
\text { training including ball games, relay races and obstacle } \\
\text { courses; tai chi; home-based walking, strengthening and } \\
\text { stretching; Fr: 3-7×/week; I: not described - only one } \\
\text { study described progression by increasing step count } \\
\text { by } 30 \% \text {; T: } 4-90 \text { minutes; D: } 10-104 \text { weeks }\end{array}$ \\
\hline
\end{tabular}

\section{Chudyk and}

Petrella $^{85}$

$\begin{array}{ll}\text { Umpierre et }^{990} & 23 \text { of } 47 \\ \text { Irvine and Taylor } & \\ & \end{array}$

Thomas et al ${ }^{89}$

14

7

Kelley and

Kelley ${ }^{87}$

Snowling and

Hopkins $^{88}$

Boulé et $\mathrm{a}^{84}$

27

HIV

O'Brien et a ${ }^{91}$
266

1513 of 8538

162 of 256

377

Both

1003

306 of 454

( 70\% M)
55.7

$55 \pm 7$

NR

Range of means 52-69

$47-68$

45-65

40-75

$18-58$

Mo: AT; Fr: most $3 \times /$ week but range of I-7×/week; I: $50 \%-85 \% \mathrm{VO}_{2} \max$ or $35 \%-85 \%$ HRmax; T: 40-75 minutes; D: 2-24 months. RT: variable

Mo: AT, RT; Fr: 2-5×/week T: AT: 30-150 minutes/ week and RT: 9-27 sets; $\mathbf{D}$ : 12-52 weeks

Mo: RT: free weights or weight machines; Fr: 2-3x/week; I: $55 \%-85 \%$ IRM or to moderate on Borg; T: 8-15 reps, I-3 sets, 5-10 exercises; D: 8-26 weeks

Mo: qi gong, RT, AT: cycle ergometer, walking cycling, skiing, swimming; Fr: 3-7x/week; I: AT: 50\%-85\% $\mathrm{VO}_{2}$ peak, $65 \%-75 \%$ HRR or $85 \%$ HRmax; T: $30-120$ minutes; if PRT, 2-3 sets of 10-12 reps;

D: 8 weeks-I year

Mo: AT: cycle ergometer, walking, jogging, cycling, swimming, skiing; Fr: $3-7 \times /$ week I: $60 \%-75 \% \mathrm{VO}_{2}$ max; T: 30-75 minutes; $\mathbf{D}$ : 10-26 weeks

Mo: AT; Fr: 3-7×/week; I: $50 \%-85 \% \mathrm{VO}_{2} \max$, HR I I0-I 40 bpm, 40\%-80\% HRR; T: 30-I20 minutes; D: 6-104 weeks.

Mo: RT; Fr: $3-5 \times /$ week; I: $50 \%-80 \%$ of I RM; T: 10-20 reps, 2-3 sets, 5-10 ex; D: 5-16 weeks Mo: AT: continuous aerobic; walking, cycling, skiing jogging, rowing, Theraband; Fr: 3-6x/week; $60 \%-75 \%$; I: $\mathrm{VO}_{2}$ max; T: 40-60 minutes; D: 8-52 weeks

Mo: AT: interval or continuous aerobic, walking, jogging, stair stepping, ski machine, stationary bike, cross country machine. Fr: $3 \times /$ week I: AT: usually $50 \%-85 \% \mathrm{VO}_{2}$ max and $60 \%-80 \%$ HRmax or RT: 60\%-80\% I RM; T: 20-60 minutes, D: 5-24 weeks 
Table 3 (Continued)

\begin{tabular}{|c|c|c|c|c|}
\hline Author & RCTs & $\mathbf{n}(\% \mathbf{M}$ or $\mathbf{F})$ & Age (years) & Characteristics of exercise intervention \\
\hline \multicolumn{5}{|c|}{ Depression/anxiety } \\
\hline Krogh et $\mathrm{al}^{93}$ & 13 & 272 & $17-85$ & $\begin{array}{l}\text { Mo: AT, RT, AT + RT; Fr: } 2-5 \times / \text { week; } \mathbf{D}: 8-16 \text { week; } \\
\text { AT: I: } 70 \%-80 \% \text { HRmax, } 50 \%-70 \% \text { aerobic capacity; } \\
\text { T: } 30-60 \text { minutes. RT: I: } 80 \% \text { RM; T: } 20 \text { minutes } \\
\text { or } 8 \text { reps } \times 3 \text { sets }\end{array}$ \\
\hline Herring et $\mathrm{al}^{94}$ & 40 & NR $(59 \% \mathrm{~F})$ & $50 \pm 10$ & $\begin{array}{l}\text { Mo: AT, RT, AT+RT; Fr: } 3 \pm \text { I } \times / \text { wk; I: variable; } \\
\text { T: } 42 \pm 22 ; \text { D: } 16 \pm 10 \text { wk }\end{array}$ \\
\hline Mead et $\mathrm{al}^{96}$ & 23 of 25 & 640 & $\geq 18$ & $\begin{array}{l}\text { Mo: AT: running, treadmill walking or walking, } \\
\text { stationary cycling; RT; Mixed: A + RT, qi gong exercises, } \\
\text { individually tailored, tai chi; D: I0 days - } 16 \text { weeks }\end{array}$ \\
\hline Rethorst et al ${ }^{95}$ & 58 & 2982 & 15-94 & $\begin{array}{l}\text { Mo: AT; Fr: } 3-5 \times / \text { week; I: moderate to high } \\
\text { T: } 20-60 \text { minutes; Mo: RT; F: 2-3 } \times / \text { week } \\
\text { T: } 20-60 \text { minutes; Mo: A + RT; T: } 20-90 \text { minutes; } \\
\text { D: acute - } 52 \text { week }\end{array}$ \\
\hline $\begin{array}{l}\text { Lawlor and } \\
\text { Hopker }^{92}\end{array}$ & II of I4 & $479(\sim 34 \% \mathrm{M})$ & All ages & $\begin{array}{l}\text { Mo: AT: running, walking; Fr: } 2-5 \times / \text { week; } \\
\text { T: } 20-60 \text { minutes; D: } 4-12 \text { week }\end{array}$ \\
\hline
\end{tabular}

Notes: In RCT columns, number of RCTs from total number of studies. In number of subject's ( $\mathrm{n}$ ) columns, number of subjects that were analyzed and total number of participants.

Abbreviations: AECOPD, acute exacerbation of COPD; AT, aerobic training; D, duration of training program; Fr, frequency; HRR, heart rate reserve; I, intensity; L/E: Lower extremity; M, male; F, female; Mo, modality; mod, moderate; NR, not reported; RT, resistance training; T, session time; U/E, upper extremity; ROM, range of motion; SWT, shuttle walking test; PRT, progressive resistance training.

AT and RT of the extremities improved maximal exercise capacity, walk distance performance, and quality of life measures in outpatient COPD compared to a control group as described by a high quality SR with an AMSTAR score of $10 .{ }^{42}$ Whether people with mild-to-moderate COPD with AECOPD benefit from exercise training is equivocal, as summarized by two SRs with AMSTAR scores of 5 and $8 .{ }^{40,44}$ Exercise training that focused on RT improved leg strength and cycling endurance as described by one review with an AMSTAR of $8 .{ }^{43}$ Unsupported and supported arm exercise showed improvement in half of the studies evaluated by one SR. ${ }^{41}$ Home exercise demonstrated improvement in exercise capacity in all studies that assessed this attribute and half of the studies that assessed quality of life. ${ }^{45}$ Dyspnea was significantly improved by aerobic and resistance trainings in two reports. ${ }^{42,44}$

Walking and cycling were the most common AT whereas free weights and Theraband ${ }^{\mathrm{TM}}$ were the most common resistance used during RT. Frequency of training ranged from twice weekly to twice daily. The intensity of aerobic training was either not described in the SRs or, in one report, was quantified as a percentage of walk test distance. ${ }^{45} \mathrm{RT}$ intensity ranged from one-third of a (repetition maximum) RM to near maximal intensity for the number of repetitions. The time for each session varied between 30 and 120 minutes, or was defined in terms of repetitions and sets for RT. ${ }^{41,43}$ Durations of studies ranged between four weeks and 18 months. There was an obvious focus on AT and RT in the SRs, and no reports described the outcomes from balance or functional training in people with COPD.

\section{Older adults}

Overall, exercise training results in improved physical and functional performance for older adults, as demonstrated by seven SRs, ${ }^{46-52}$ although initial health status influenced both the ability to participate in certain programs as well as the outcomes of the training. Most SRs and many RCTs included a mix of RT, BT, and functional training rather than focusing on a single type of training. Thus, evidence for the most part is provided from multifaceted training rather than a singular approach. Many other aspects of the training varied widely, with a training frequency most often of two to three times a week, total duration that ranged from 2 to 104 weeks, and a variable intensity level (Table 3). SRs, which had AMSTAR scores ranging from 5 to 11 , showed that mixed training improved balance ${ }^{47,49,52}$ and strength, ${ }^{47,51,52}$ reduced falls, ${ }^{48}$ and improved functional measures such as the 6-minute walk distance (6MWD). ${ }^{46,49,51,52}$ An SR that had a singular focus on RT with an AMSTAR of 11 showed widespread improvements in lower extremity strength, aerobic capacity, and several physical performance tests (Table 4) ${ }^{50}$ In addition, this SR provided evidence that RT reduced the relative risk of death and pain. ${ }^{50}$

\section{Heart failure}

Six meta-analyses ${ }^{53-58}$ investigated the benefits of AT and/or RT in patients with chronic heart failure. Most of these papers 


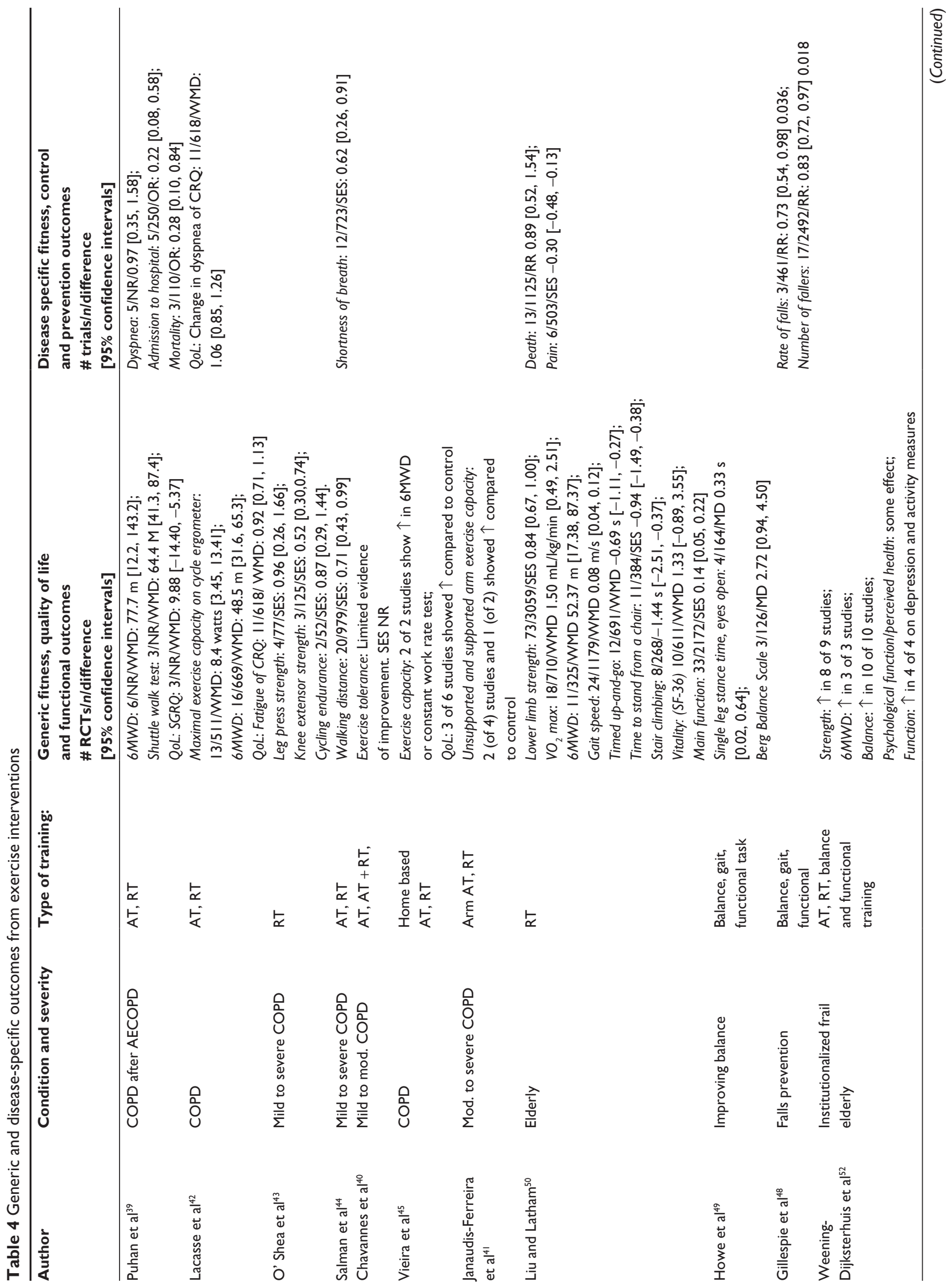




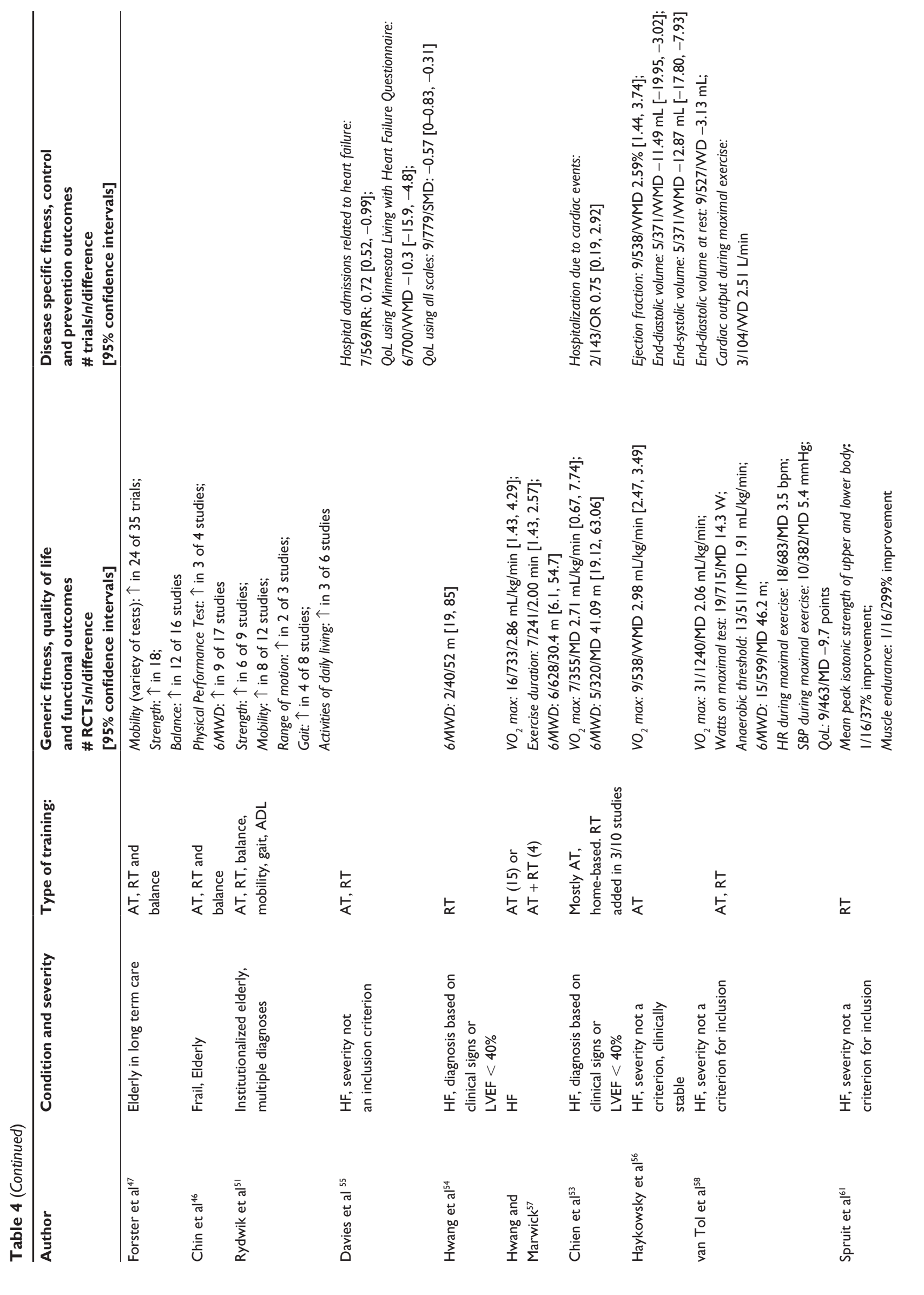



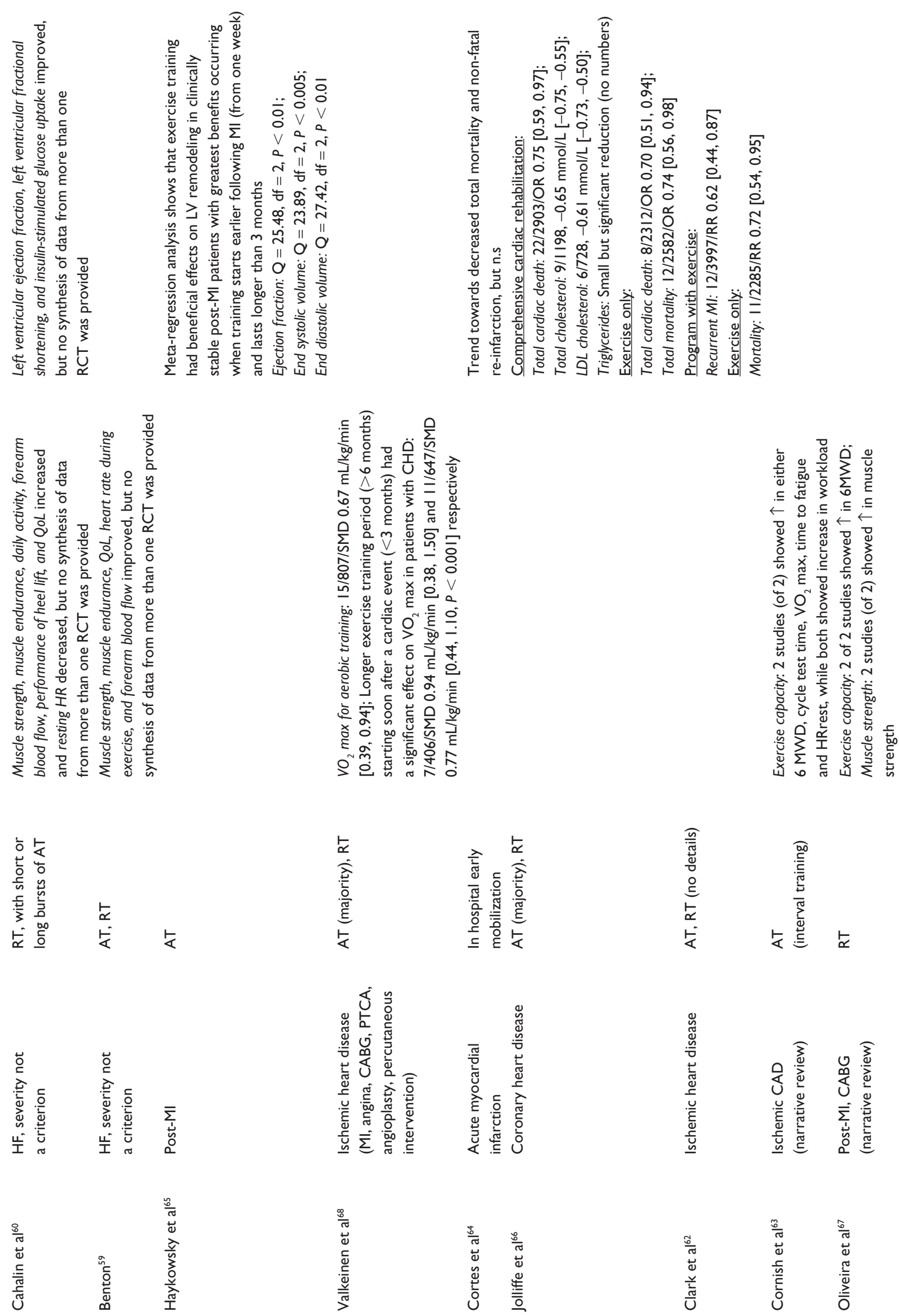


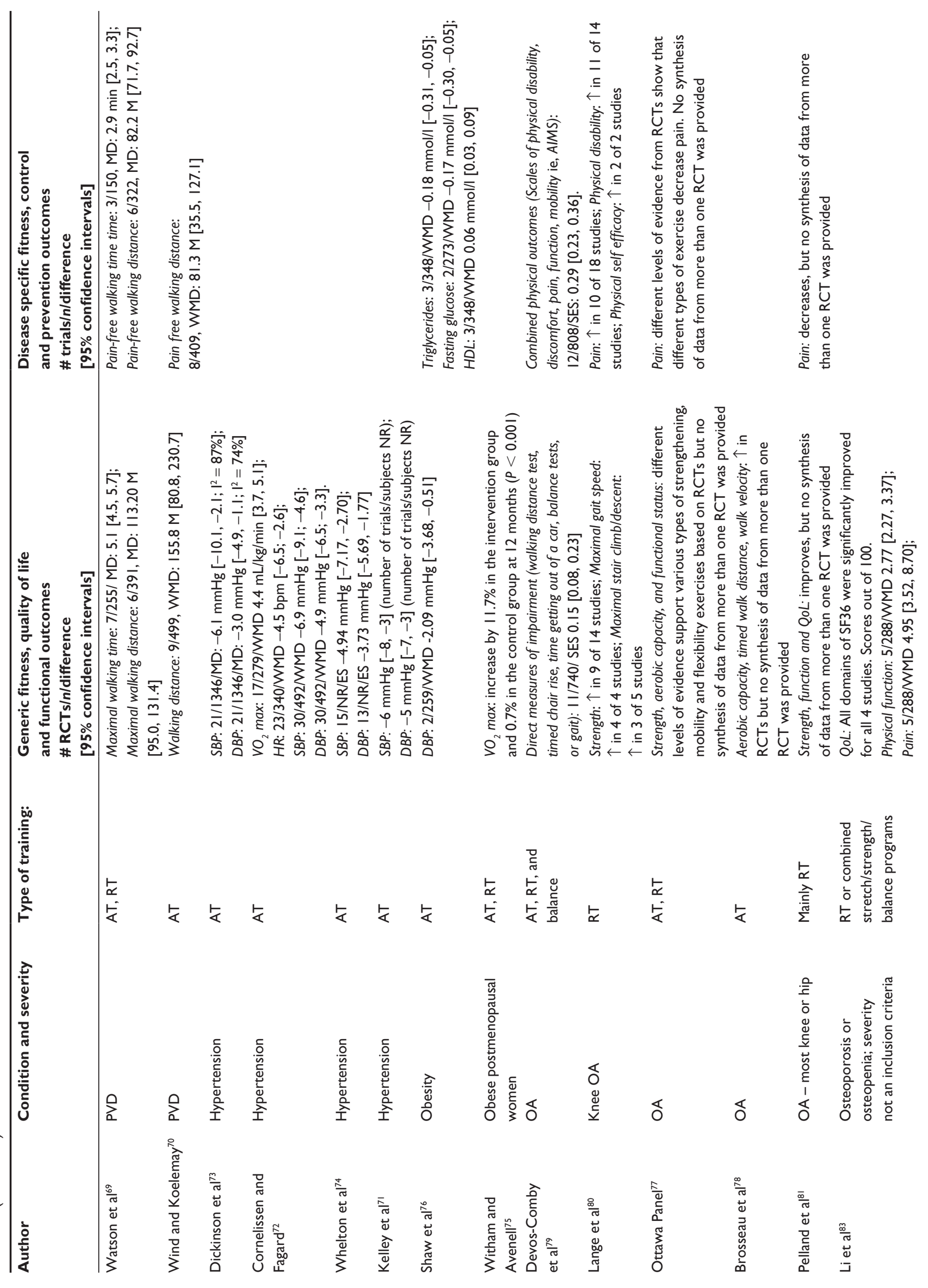



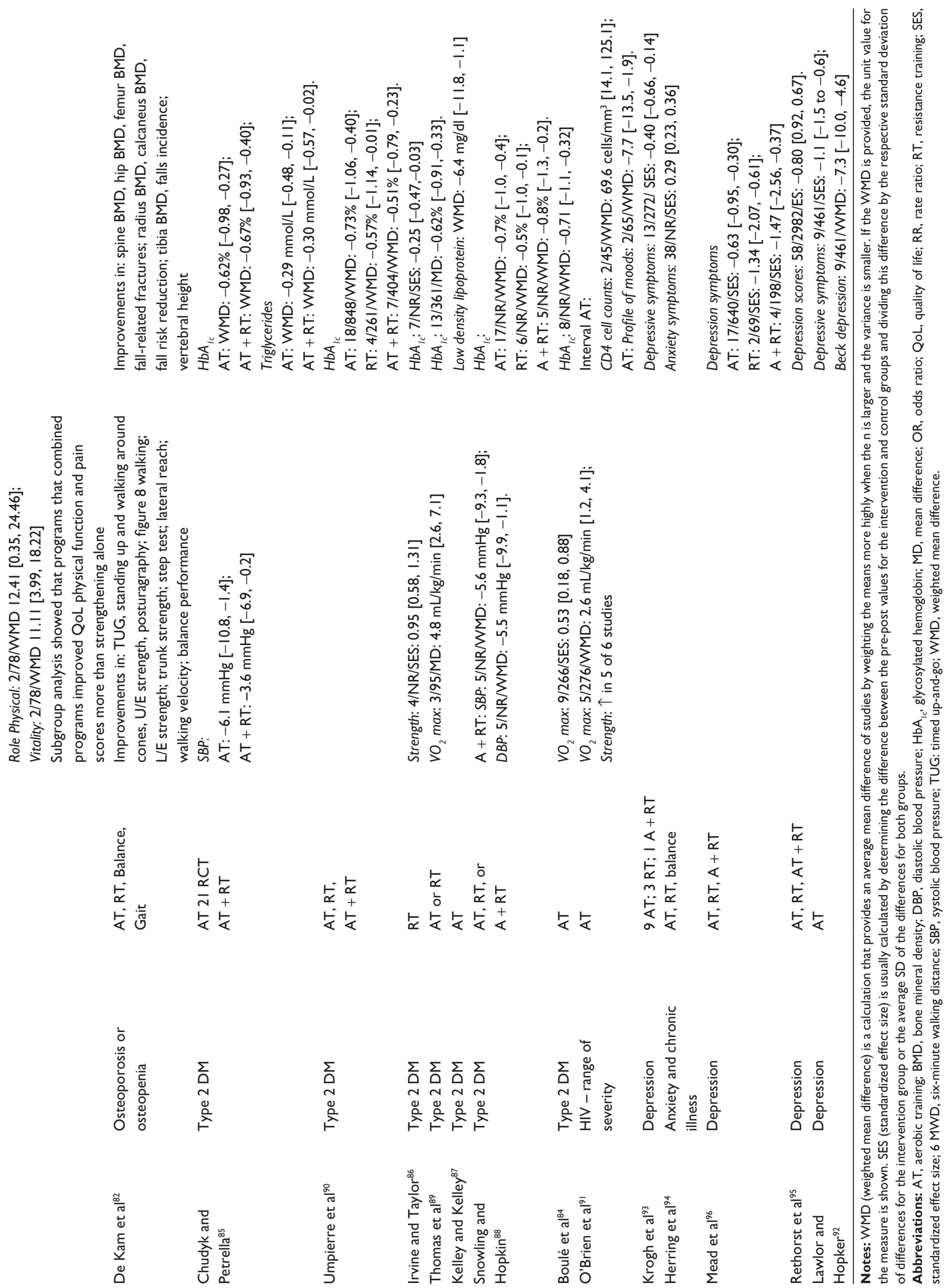
had AMSTAR scores ranging from 6 to 8 , although one ${ }^{57}$ had a score of 3 . Based on these papers, it can be concluded that AT and RT significantly improved numerous cardiopulmonary exercise outcomes, including increasing $\mathrm{VO}_{2}$ max, 6MWD, maximal workload, and anaerobic threshold. It also improved several cardiac system factors, including increasing the heart rate and cardiac output during maximal exercise, improving left ventricular ejection fraction, and the end-diastolic and end-systolic ventricular volume. Two metaanalyses also reported that exercise significantly improved quality of life in these patients. ${ }^{55,58}$

There were no meta-analyses that investigated the role of AT and/or RT on muscle strength or endurance. Three reviews without meta-analyses investigated the benefits of exercise on a large number of outcomes, including several that are specific to muscle performance. ${ }^{59-61}$ Exercise improved upper and lower extremity strength and muscle endurance, increased oxidative enzyme activity in the quadriceps muscle, and increased forearm blood flow. However, these results must be interpreted with caution as these reports were SRs without meta-analyses, and the AMSTAR scores were relatively low (2 to 6), with few details quantifying the magnitude of the effect.

The AT was relatively consistent across all the studies, in terms of frequency, mode, and intensity. Subjects exercised 2-7 days per week, with most trials within each SR providing the exercise intervention for 3 days per week. Cycle ergometry and treadmill walking were the most commonly used modalities. Most studies prescribed an AT intensity based on a maximal cardiopulmonary exercise test, with exercise intensities ranging from $50 \%$ to $80 \%$ of $\mathrm{VO}_{2}$ max. The time per session and the durations of the programs were very variable, ranging from 12 to 100 minutes and 1 to 14 months, respectively. Where described, the resistance training prescription tended to be based on $40 \%$ to $80 \%$ of one RM.

\section{Ischemic heart disease}

Seven SRs investigated the benefits of early mobilization, anaerobic training, and/or RT in ischemic heart disease patients. ${ }^{62-68}$ The AMSTAR scores ranged from 3 to 10, with two reviews having a score of $3,{ }^{63,67}$ and one a score of $5 .{ }^{68}$ The remaining reviews had AMSTAR scores exceeding 8 .

The study populations were predominantly male. There was variation in the specific type of intervention (both AT and RT), and differing endpoints were utilized to evaluate benefit and responses. Five reviews examined the effects of $\mathrm{AT},{ }^{62,63,65,66,68}$ three reported the effects of both AT and
$\mathrm{RT}^{62,66,68}$ one examined the sole effect of early in-hospital mobilization, ${ }^{64}$ and one examined the sole effect of RT. ${ }^{67}$ Some work focused on reporting disease-specific outcomes (ie, recurrent myocardial infarction, cholesterol and triglyceride levels, ejection fraction, and end-systolic and enddiastolic volumes), ${ }^{62,64,65,66}$ while others studied indices of overall activity and fitness (ie, walking distance, maximal aerobic capacity, exercise duration, and muscle strength) $)^{63,67,68}$ in these populations with ischemic heart disease. Quality of life and health care utilization were not studied or specifically reported, and significant variation was evident in the duration of follow-up.

Early in-hospital mobilization demonstrated nonsignificant trends towards decreased total mortality and nonfatal re-infarction. ${ }^{64}$ The results suggest that anaerobic and RT reduces the risk of total cardiac death, recurrent myocardial infarction, as well as cholesterol and triglyceride levels. Improvements were also demonstrated in peak oxygen uptake, exercise duration, exercise workload, and 6MWD. The findings suggest that benefits were greater when exercise programming was initiated soon after an ischemic event (as early as 1 week post-myocardial infarction), and when exercise program duration exceeded either 3 or 6 months (rather than $<3$ months).

\section{Peripheral vascular disease}

Exercise training significantly improved the walking distance and pain-free walking distance in patients with peripheral vascular disease (PVD) when compared to standard care as evidenced by two meta-analyses with relatively high AMSTAR scores of 9 and 7. ${ }^{69,70}$ Exercise training was generally supervised. The optimal intensity was not clear, although it was suggested that patients exercised until near maximal pain. The majority of the studies applied an exercise regimen of two to three times per week for 30-60 minutes, with these usually incorporating walking, leg exercises, or treadmill training. Some encouraged additional home exercise.

\section{Hypertension}

Exercise training (mainly aerobic exercise) significantly lowered the systolic (SBP) and diastolic blood pressure (DBP) in people with hypertension, as supported by four meta-analyses. ${ }^{71-74}$ Two of the SRs ${ }^{73,74}$ had AMSTAR scores of 8 of 11 while two others had scores of 7 and $4 .{ }^{71,72} \mathrm{VO}_{2}$ max increased significantly and resting heart rate (HR) decreased after exercise training. ${ }^{72}$ Exercise training also demonstrated significant improvement in cardiovascular risk factors as shown by an increase in high density lipoprotein 
cholesterol, and decreases in glucose, insulin levels, and the homeostatic model assessment index (a measure of insulin resistance), despite the inclusion of normotensives in the analysis. ${ }^{72}$ Reductions were greater for SBP in AfricanAmerican individuals and for DBP in Asian individuals, but only a small number of trials were conducted in people of different ethnic backgrounds. ${ }^{74}$

A variety of study designs were included in the metaanalyses, but the majority of the studies included training programs that involved mainly aerobic exercise (walking, jogging, cycling), usually three to five times per week, for 30 to 60 minutes, at moderate intensity. According to two SRs there is no relationship between blood pressure (BP) response and training characteristics (intensity, frequency), except for a lesser BP reduction associated with a longer total trial duration, probably due to loss of compliance in long trials. ${ }^{72,74}$

Because aerobic exercise also showed positive effects in normotensives, these data suggest that aerobic exercise is an important strategy not only in treating high blood pressure, but could potentially be used as prophylaxis. ${ }^{74}$

\section{Obesity}

Data on the effect of physical activity on fitness outcomes in this population were limited. In post-menopausal obese women, an exercise-based program improved $\mathrm{VO}_{2}$ max as shown in one RCT reported by one SR. ${ }^{75}$ A Cochrane review with an AMSTAR score of 11 did not report the effect of physical activity on $\mathrm{VO}_{2}$ max but found a decrease in DBP, reduced triglycerides and glucose, and increased HDL levels. ${ }^{76}$ The majority of trials in the two SRs consisted of AT, usually two to three times per week, at $60 \%-80 \%$ of $\mathrm{VO}_{2}$ max or HRmax, with different durations utilized.

\section{Osteoarthritis}

Results from the five SRs examining the effects of exercise on patients with knee or hip OA consistently show beneficial results for fitness levels and functional performance, though emphasis was primarily on disease-specific outcomes such as joint pain or physical dysfunction. ${ }^{77-81}$ AMSTAR scores were between 7 and 9, and most studies failed to report publication bias or conflicts of interest.

Isotonic RT targeting the quadriceps or hamstrings muscle groups was the most common exercise intervention, though AT and range of motion exercises were also included. Control interventions primarily included patient education or usual care with no additional treatment. Most exercise interventions lasted 3-6 months, though some were as short as 4 weeks and as long as 24 months. Some studies required daily exercise, while others included only two or three sessions per week. Individual sessions were typically 45-60 minutes in length and complied with usual dosage characteristics for exercise in healthy populations (ie, three sets of ten repetitions for resistance training, and $50 \%$ to $80 \%$ of maximum heart rate for aerobic interventions).

Pain and physical function were consistently improved following exercise, but no particular type of exercise was found to be superior. Studies examining RT found improvements in strength compared to control interventions, ${ }^{77,81}$ while studies examining the effects of AT found improvements in aerobic capacity, walking speed, and walking distance. ${ }^{77-80}$ All studies found exercise to be a safe and feasible intervention with minimal side effects for patients with OA.

\section{Osteoporosis}

Most of the SRs identified in this study investigated the benefits of exercise in the prevention of osteoporosis or osteopenia in healthy, pre- or post-menopausal women. Two SRs investigated the impact of exercise in women diagnosed with osteoporosis or osteopenia, and these had AMSTAR scores of $7^{82}$ and $6^{83}$, indicating moderate quality overall. One of these was an SR without a meta-analysis that evaluated the benefits of AT, RT, BT, and gait on numerous exercise- and disease-related outcomes. It was reported that exercise resulted in improvements in balance measures such as the timed-up-and-go, upper extremity strength, and walking velocity, as well a reduction in fall incidence and fall-related fractures. ${ }^{82}$ The other paper included a meta-analysis investigating the benefits of RT with or without balance exercise, and reported that exercise improved several quality-of-life domains including physical function, pain, and vitality. ${ }^{83}$

\section{Type 2 diabetes mellitus}

Seven meta-analyses investigated the benefits of RT and or AT in people with type 2 diabetes with AMSTAR scores that ranged from 6 to $11 .^{84-90}$ Aerobic exercise improved $\mathrm{VO}_{2}$ max as evidenced by two SRs with AMSTAR scores of 6 and 11.84,89 Two SRs with AMSTAR scores of 6 also demonstrated reductions in SBP and DBP after AT, RT, or a combination of AT and RT. ${ }^{85,88}$ Strength measures improved after RT, as described by one SR with an AMSTAR of $7 .{ }^{86}$ Glycosylated hemoglobin $\left(\mathrm{HbA}_{1 \mathrm{c}}\right)$, a measure of blood sugar levels, was the most consistent outcome reported in all of the included SRs, with a reduction in this seen in all reviews regardless of the type of exercise training. ${ }^{84-90}$ 
The exercise interventions described were quite varied across the SRs. AT consisted of stationary cycling, outdoor cycling, walking, jogging, swimming, rowing, or skiing. RT was performed using a Theraband, free weights, or weight machines. AT intensity ranged $50 \%-85 \%$ of $\mathrm{VO}_{2}$ max, $35 \%-85 \%$ of HRmax, or $40 \%-80 \%$ of HRR. RT intensity ranged $50 \%-85 \%$ of one RM. The exercise sessions were between 30 and 120 minutes, and the duration of the program ranged from 5 to 52 weeks. The SRs primarily focused on the benefits of AT, RT, or a combination of these interventions. However, no studies focused on balance or functional training, or reported functional or quality-of-life outcomes.

\section{HIVIAIDS}

Interval and continuous AT improved $\mathrm{VO}_{2}$ max in patients living with HIV, as evidenced by a Cochrane review with an AMSTAR score of $10 .{ }^{11}$ This SR also described improved strength in five of the six studies that reported this measure. Additional benefits from exercise training include no change in the viral load, an increase in CD4 cell counts after interval AT, and improved Profile of Moods scores after AT. A summary of the interventions applied is shown in Table 3.

\section{Depression}

The five SRs relating to depression were moderate-to-high quality (AMSTAR 8-11). ${ }^{92-96}$ Exercise programs (AT, RT, or a combination of these) for people diagnosed with major or minor depression were found to result in improved scores on depression and mood, particularly in the short term..$^{92,93}$ Medium- to long-term effects were less clear. ${ }^{92,93}$ As a group of studies, the interventions were not well described. Most examined both AT, RT, and mixed AT plus RT, except one ${ }^{92}$ that focused on AT. The intensity of AT, if described, was moderate to high. ${ }^{93,95}$ Each session was 30 to 60 minutes in length, with these performed one to five times per week, and the duration of the programs was between 10 days and 52 weeks.

\section{Discussion}

This synthesis examined 58 systematic reviews of exercise training in people with AECOPD, COPD, or eleven chronic conditions commonly associated with COPD. Overall, this review provides Level $1 \mathrm{~A}$ evidence ${ }^{97}$ that exercise training improves generic or disease-specific measures of fitness in several disease populations. Markers of endurance (aerobic capacity, 6MWD) were shown to improve in all conditions except depression, whereas strength was shown to improve in most conditions with the exception of PVD, hypertension, obesity, and depression. In addition, several studies in different disease populations also reported improvements in quality of life, function, control, or prevention outcomes. Depression was the only condition where SRs exclusively focused on disease-specific outcomes (measures of depression and anxiety) ${ }^{92-96}$ Exercise training also decreases the risk of mortality in older adults, ${ }^{50}$ and in those with $\mathrm{COPD}^{39}$ or ischemic heart disease. ${ }^{66}$

Overwhelmingly, the most common types of training provided to people with all conditions reviewed were AT and RT. Balance and functional training were less commonly applied SRs of exercise training. However, these interventions had widespread application in most SRs that examined older adults, ${ }^{46-49,51,52}$ and in one SR that examined OA patients ${ }^{79}$ and one that examined osteoporosis patients. ${ }^{82}$

The quality of the SRs for most conditions was moderate to excellent $(>65 \%)$ as evaluated by AMSTAR scores. However, SRs that examined training outcomes in heart failure, ischemic heart disease, hypertension, and osteoporosis scored moderately poorly on the AMSTAR ( $\geq 51 \%$ but $\leq 61 \%$ ). The listing of articles that were excluded from in the review was the most common item omitted from most SRs, which may have been due to page constraints. Even with this item excluded, the AMSTAR scores remained relatively low for heart failure (56\%) whereas scores for the other conditions were $\geq 66 \%$. In addition to the SR design and strength of AMSTAR scores, the number of participants and RCTs for each condition, including COPD, were large. These numbers further strengthen the foundation of evidence and recommendations for exercise training as an intervention that produces positive outcomes for COPD and commonly associated conditions. ${ }^{98}$

Although COPD patients often have one or more comorbidities, no information is available, on prescription parameters of exercise training for patients with multimorbidity. Training parameters from studies examining conditions that are common comorbidities might be applicable, however, consideration should be give to similarities of patient demographics and details of training, especially intensity. Given that the most common conditions associated with COPD are obesity (prevalence of $54 \%$ ), ${ }^{26}$ osteoporosis $(60 \%-70 \%)^{23}$ and osteoarthritis (increasing prevalence to $40 \%$ at age 75$)^{27}$ (see Table 1), data from SRs describing these conditions may have the greatest relevance. The pertinence of these data is further strengthened by the similarity in age of participants from SRs that examined exercise training in people who were obese, had osteoporosis or osteoarthritis to those affected by COPD. In contrast, subjects were somewhat younger in 
some of the RCTs that examined obesity. Gender was mixed in all of these conditions, except in the case of the SRs on osteoporosis in which predominantly women were studied. ${ }^{82,83}$

The modalities of AT and RT for obesity, osteoporosis, and osteoarthritis were similar to those typically applied for COPD patients, although exercise training for these conditions utilized more diverse types of training. The intensity of AT appeared to be much higher for obese individuals and those with arthritis compared to that prescribed for COPD patients; intensity was commonly based on aerobic or HR parameters for the former compared to walk distance or dyspnea measures used in COPD patients. This raises the notion that a broader spectrum approach to exercise training in order to improve fitness in patients with COPD and comorbidities such as obesity, osteoporosis, and osteoarthritis may be effective. However, exercise training of the more complex COPD patient (with multiple comorbidities) may result in smaller gains of disease-specific outcomes due to the inability to achieve comparable AT and RT intensities (compared to those achieved in patients with a single condition). These issues merit further study.

Because obesity is a major cause of morbidity and a significant risk factor for other comorbidities such as heart disease and diabetes, ${ }^{26,75,76} \mathrm{SRs}$ addressing exercise fitness outcomes other than body composition are needed in order to determine the overall impact of exercise training in this particular group. Although altering body composition may be the primary outcome for people who are obese, understanding the training and physiologic determinants to achieve that goal are essential; thus fitness outcomes such as aerobic capacity and strength measures should be reported in order to better evaluate the type and intensity of training that results in improvement. Moreover, it has also recently been demonstrated that some obese COPD patients have better fitness outcomes than non-obese COPD patients. ${ }^{99}$ Determining how physical activity interventions can attenuate obesity, reduce cardiovascular risk factors, and improve overall fitness in people with COPD needs further attention.

Depression is a common comorbidity in COPD with estimates of prevalence ranging between $10 \%$ and $80 \%{ }^{25}$ SRs that examined the effect of exercise on depression had participants with an age range similar to those with COPD, and the interventions were similar to many of the other conditions in this review. A major contrast to the other SRs was that only disease-specific outcomes and no generic fitness measures were reported. All five SRs demonstrated significant decreases in depression or anxiety scores. ${ }^{92-96}$ These benefits were reported regardless of whether AT, RT, or a combination of AT and RT were applied. Of interest is the finding that the benefits decreased in response to longer training programs. More RCTs are needed to examine clinical sub-groups (minor, moderate, major depression), to compare different forms of exercise, and to clarify the appropriate dose/intensity of physical exercise in addition to the mechanism of improvement. Designing a training program that might result in gains related to self-efficacy would be very different from a regimen that requires improved aerobic fitness or an endorphin response to induce a therapeutic benefit. A better understanding of the type of depression most commonly experienced by COPD will also provide further insight into if and how exercise training can reduce the clinical severity of these two conditions.

Exercise training for older adults showed the greatest contrasts to interventions applied to COPD and other associated conditions in this review. This is especially important given that many individuals with COPD who present for health care interventions are 65 or older. Major foci appeared to be on balance and functional training rather than predetermined intensities of AT or RT. ${ }^{46-49,51,52}$ In addition, frequently reported outcomes by the SRs on the elderly included balance/falls, and physical performance measures that were functional (ie, timed up-and-go, stair climbing) as opposed to more physiologic measures of strength and endurance. Exercise training for older adults may provide an excellent model to further broaden exercise prescription for COPD patients because, as with the elderly, ${ }^{48,49}$ people with COPD have a high relative risk of falls. ${ }^{100}$ In addition, the physical activity level of COPD patients is often comparable to much older, healthier adults. Thus, a greater focus on balance and functional training may not only reduce falls in COPD patients but might also result in greater gains in daily physical activity outside of pulmonary rehabilitation. ${ }^{101}$

\section{Limitations}

Although this synthesis of systematic reviews examined several conditions commonly associated with COPD, the participants within each of the SRs most often had a homogeneous presentation of the condition of interest. Only SRs of the elderly appeared to include participants with diverse and multiple morbidities. For this reason, it might be challenging to apply modalities in a sufficient dose in order to induce both generic and disease-specific benefits that address a complex patient with COPD and multimorbidities. The more functional approach of physical activity training, even in the frail elderly, did result in functional gains, and this approach may also prove to be beneficial to the AECOPD patients with multimorbidities. 
Many SRs included lengthy training programs but did not compare the benefits of shorter versus longer programs, with the exception of SRs that evaluated those with hypertension $^{72,74}$ and depression. ${ }^{92,93}$ These SRs indicated a slightly diminished response with longer training programs, which may have resulted in a blunting of the physiologic benefits of exercise, decreased adherence to the exercise training program, or a continued onslaught of inciting factors that could no longer be countered by the benefits of exercise training.

\section{Conclusions}

In summary, this systematic review showed evidence of significant benefits from exercise training in AECOPD, $\mathrm{COPD}$, and many conditions that are common comorbidities associated with COPD. Exercise training, primarily consisting of AT and/or RT, leads to meaningful generic and disease-specific benefits in these conditions. Meta-analyses of endurance (aerobic or exercise capacity, 6MWD) were shown to improve in most conditions whereas metaanalyses of strength were shown to improve in five of the 13 conditions (that is, COPD, older adults, heart failure, ischemic heart disease, and diabetes). Additional research is required to determine the added benefit of RT plus AT in several conditions that are common comorbidities associated with COPD.

A broader approach to exercise and activity prescription in pulmonary rehabilitation for AECOPD may induce therapeutic benefits to ameliorate clinical sequelae associated with COPD and comorbidities. For example, BT and functional training, similar to that applied in older adults, could potentially be very effective in improving functional outcomes including fall risk, and may have a greater impact on daily physical activity than recently demonstrated. ${ }^{101}$

\section{Disclosure}

The authors report no conflicts of interest in this work.

\section{References}

1. World Health Organization. Programmes and projects: Chronic Respiratory Diseases: Burden of COPD Web page. Geneva, Switzerland: World Health Organization; 2011. Available from: http://www.who.int/ respiratory/copd/burden/en/index.html. Accessed October 5, 2011.

2. de Melo MN, Ernst P, Suissa S. Rates and patterns of chronic obstructive pulmonary disease exacerbations. Can Respir J. 2004;11(8):559-564.

3. Vestbo J. Clinical assessment, staging, and epidemiology of chronic obstructive pulmonary disease exacerbations. Proc Am Thorac Soc. 2006;3(3):252-256.

4. Fitzgerald JM, Haddon JM, Bradley-Kennedy C, Kuramoto L, Ford GT; RUSIC Study Group. Resource use study in COPD (RUSIC): a prospective study to quantify the effects of COPD exacerbations on health care resource use among COPD patients. Can Respir J. 2007;14(3):145-152.
5. Mittmann N, Kuramoto L, Seung SJ, Haddon JM, Bradley-Kennedy C, Fitzgerald JM. The cost of moderate and severe COPD exacerbations to the Canadian healthcare system. Respir Med. 2008;102(3): 413-421.

6. Sin DD, Tu JV. Are elderly patients with obstructive airway disease being prematurely discharged? Am J Respir Crit Care Med. 2000;161(5): $1513-1517$.

7. Spruit MA, Gosselink R, Troosters T, et al. Muscle force during an acute exacerbation in hospitalised patients with COPD and its relationship with CXCL8 and IGF-I. Thorax. 2003;58(9):752-756.

8. Gillick MR, Serrell NA, Gillick LS. Adverse consequences of hospitalization in the elderly. Soc Sci Med. 1982;16(10):1033-1038.

9. Hirsch CH, Sommers L, Olsen A, Mullen L, Winograd CH. The natural history of functional morbidity in hospitalized older patients. $J \mathrm{Am}$ Geriatr Soc. 1990;38(12):1296-1303.

10. Inouye SK, Wagner DR, Acampora D, Horwitz RI, Cooney LM Jr, Tinetii ME. A controlled trial of a nursing-centered intervention in hospitalized elderly medical patients: the Yale Geriatric Care Program. J Am Geriatr Soc. 1993;41(12):1353-1360.

11. Sager MA, Franke T, Inouye SK, et al. Functional outcomes of acute medical illness and hospitalization in older persons. Arch Intern Med. 1996;156(6):645-652.

12. Mahoney JE, Sager MA, Jalaluddin M. New walking dependence associated with hospitalization for acute medical illness: incidence and significance. J Gerontol A Biol Sci Med Sci. 1998;53A(4): M307-M312.

13. Pitta F, Troosters T, Probst VS, Spruit MA, Decramer M, Gosselink R. Physical activity and hospitalization for exacerbation of COPD. Chest. 2006;129(3):536-544.

14. World Health Organization. Facing the Facts: The Impact of Chronic Disease in Canada. Geneva, Switzerland: World Health Organization; 2011. Available from: http://www.who.int/chp/chronic_disease_report/ media/CANADA.pdf. Accessed November 21, 2011.

15. Wild S, Roglic G, Green A, Sicree R, King H. Global prevalence of diabetes: estimates for the year 2000 and projections for 2030. Diabetes Care. 2004;27(5):1047-1053.

16. World Diabetes Foundation. Diabetes Facts. Web page. Gentofte, Denmark: World Diabetes Foundation [updated May 5, 2011]. Available from: http://www.worlddiabetesfoundation.org/composite-35.htm. Accessed October 6, 2011.

17. Young AS, Klap R, Shoai R, Wells KB. Persistent depression and anxiety in the United States: prevalence and quality of care. Psychiatr Serv. 2008;59(12):1391-1398.

18. Tu K, Chen Z, Lipscome LL, Canadian Hypertension Education Program Outcomes Research Taskforce. Prevalence and incidence of hypertension from 1995-2005: a population-based study. CMAJ. 2008;178(11):1429-1435.

19. Hajjar I, Kotchen TA. Trends in prevalence, awareness, treatment, and control of hypertension in the United States, 1988-2000. JAMA. 2003;290(2):199-206.

20. Selvin E, Erlinger TP. Prevalence of and risk factors for peripheral arterial disease in the United States: results from the National Health and Nutrition Examination Survey, 1999-2000. Circulation. 2004;110(6):738-743.

21. International Osteoporosis Foundation. Facts and Statistics about Osteoporosis and its Impact. Available from: http://www.iofbonehealth. org/facts-and-statistics.html\#factsheet-category-23. Accessed February 29, 2012.

22. Ho KK, Anderson KM, Kannel WB, Grossman W, Levy D. Survival after the onset of congestive heart failure in Framingham Heart Study subjects. Circulation. 1993;88(1):107-115.

23. Kopec JA, Rahman MM, Berthelot JM, et al. Descriptive epidemiology of osteoarthritis in British Columbia, Canada. J Rheumatol. 2007;34(2);386-393.

24. Rutten FH, Cramer MJ, Lammers JW, Grobbee DE, Hoes AW. Heart failure and chronic obstructive pulmonary disease: an ignored combination? Eur J Heart Fail. 2006;8(7):706-711. 
25. Barnes PJ, Celli BR. Systemic manifestations and comorbidities of COPD. Eur Respir J. 2009;33(5):1165-1185.

26. Eisner MD, Blanc PD, Sidney S, et al. Body composition and functional limitation in COPD. Respir Res. 2007;8:7

27. Ferguson GT, Claverley PM, Anderson JA, et al. Prevalence and progression of osteoporosis in patients with COPD: results from the TOwards a Revolution in COPD Health Study. Chest. 2009;136(6):1456-1465.

28. Smidt N, de Vet HC, Bouter LM, et al. Effectiveness of exercise therapy: a best-evidence summary of systematic reviews. Aust $J$ Physiother 2005;51(2):71-85.

29. Kujala UM. Evidence on the effects of exercise therapy in the treatment of chronic disease. Br J Sports Med. 2009;43(8):550-555.

30. American College of Sports Medicine. ACSM's Guidelines for Exercise Testing and Prescription. 8th ed. Philadelphia, PA: Lippincott Williams and Wilkins, 2009.

31. Canadian Society for Exercise Physiology. Canadian Physical Activity Guidelines: Clinical Practice Guideline Development Report. Ottawa, Canada: Canadian Society for Exercise Physiology; 2011. Available from: http://www.csep.ca/CMFiles/Guidelines/CPAGuideline_Report_ JAN2011.pdf. Accessed October 14, 2011.

32. Lemmens V, Oenema A, Knut IK, Brug J. Effectiveness of smoking cessation interventions among adults: a systematic review of reviews. Eur J Cancer Prev. 2008;17(6):535-544.

33. Ernst E. A systematic review of systematic reviews of homeopathy. Br J Clin Pharmacol. 2002;54(6):577-582.

34. Kujala UM. Evidence for exercise therapy in the treatment of chronic disease based on at least three randomized controlled trials summary of published systematic reviews. Scand J Med Sci Sports. 2004;14(6):339-345.

35. Greaves CJ, Sheppard KE, Abraham C, et al. Systematic review of reviews of intervention components associated with increased effectiveness in dietary and physical activity interventions. BMC Public Health. 2011;11:119

36. Taylor NF, Dodd KJ, Damiano DL. Progressive resistance exercise in physical therapy: a summary of systematic reviews. Phys Ther. 2005;85(11):1208-1223.

37. The Cochrane Collaboration. Cochrane Handbook for Systematic Reviews of Interventions. Version 5.1.0 [updated March 2011] Higgins JPT, Green S, editors. Available from: http://www.cochranehandbook.org/. Accessed February 29, 2012.

38. Shea BJ, Hamel C, Wells GA, et al. AMSTAR is a reliable and valid measurement tool to assess the methodological quality of systematic reviews. J Clin Epidemiol. 2009;62(10):1013-1020.

39. Puhan MA, Scharplatz M, Troosters T, Walters EH, Steurer J. Pulmonary rehabilitation following exacerbations of chronic obstructive pulmonary disease. Cochrane Database Syst Rev. 2009;(1):CD005305.

40. Chavannes N, Vollenberg JJ, van Schayck CP, Wouters EF. Effects of physical activity in mild to moderate COPD: a systematic review. Br J Gen Pract. 2002;52(480):574-578.

41. Janaudis-Ferreira T, Hill K, Goldstein R, Wadell K, Brooks D. Arm exercise training in patients with chronic obstructive pulmonary disease: as systematic review. J Cardiopulm Rehabil Prev. 2009;29(5): 277-283.

42. Lacasse Y, Goldstein R, Lasserson TJ, Martin S. Pulmonary rehabilitation for chronic obstructive pulmonary disease. Cochrane Database Syst Rev. 2006;(4):CD003793.

43. O'Shea SD, Taylor NF, Paratz JD. Progressive resistance exercise improves muscle strength and may improve elements of performance of daily activities for people with COPD: a systematic review. Chest. 2009;136(5):1269-1283.

44. Salman GF, Mosier MC, Beasley BW, Calkins DR. Rehabilitation for patients with chronic obstructive pulmonary disease: meta-analysis of randomized controlled trials. J Gen Intern Med. 2003;18(3): 213-221.

45. Vieira DS, Maltais F, Bourbeau J. Home-based pulmonary rehabilitation in chronic obstructive pulmonary disease patients. Curr Opin Pulm Med. 2010;16(2):134-143.
46. Chin A Paw MJ, van Uffelen JG, Riphagen I, van Mechelen W. The functional effects of physical exercise training in frail older people: a systematic review. Sports Med. 2008;38(9):781-793.

47. Forster A, Lambley R, Young JB. Is physical rehabilitation for older people in long-term care effective? Findings from a systematic review. Age Ageing. 2010;39(2):169-175.

48. Gillespie LD, Gillespie WJ, Robertson MC, Lamb SE, Cumming RG, Rowe BH. Interventions for preventing falls in elderly people. Cochrane Database Syst Rev. 2003;(4):CD000340.

49. Howe TE, Rochester L, Jackson A, Banks PM, Blair VA. Exercise for improving balance in older people. Cochrane Database Syst Rev. 2008;(4):CD004963.

50. Liu CJ, Latham NK. Progressive resistance strength training for improving physical function in older adults. Cochrane Database Syst Rev. 2009;(3):CD002759.

51. Rydwik E, Frändin K, Akner G. Effects of physical training on physical performance in institutionalised elderly patients (70+) with multiple diagnoses. Age Ageing. 2004;33(1):13-23.

52. Weening-Dijksterhuis E, de Greef MH, Scherder EJ, Slaets JP, van der Schans CP. Frail institutionalized older persons: a comprehensive review on physical exercise, physical fitness, activities of daily living, and quality-of-life. Am J Phys Med Rehabil. 2011;90(2):156-168.

53. Chien CL, Lee CM, Wu YW, Chen TA, Wu YT. Home-based exercise increases exercise capacity but not quality of life in people with chronic heart failure: a systematic review. Aust $J$ Physiother. 2008;54(2);87-93.

54. Hwang CL, Chien CL, Wu YT. Resistance training increases 6-minute walk distance in people with chronic heart failure: a systematic review. J Physiother. 2010;56(2):87-96.

55. Davies EJ, Moxham T, Rees K, et al. Exercise training for systolic heart failure: Cochrane systematic review and meta-analysis. Eur $J$ Heart Fail. 2010;12(7):706-715.

56. Haykowsky MJ, Liang Y, Pechter D, Jones LW, McAlister FA, Clark AM. A meta-analysis of the effect of exercise training on left ventricular remodeling in heart failure patients: the benefit depends on the type of training performed. J Am Coll Cardiol. 2007;49(24):2329-2336.

57. Hwang R, Marwick T. Efficacy of home-based exercise programmes for people with chronic heart failure: a meta-analysis. Eur J Cardiovasc Prev Rehabil. 2009;16(5):527-535.

58. van Tol BA, Huijsmans RJ, Kroon DW, Schothorst M, Kwakkel G. Effects of exercise training on cardiac performance, exercise capacity and quality of life in patients with heart failure: a meta-analysis. Eur $J$ Heart Fail. 2006;8(8):841-850.

59. Benton MJ. Safety and efficacy of resistance training in patients with chronic heart failure: research-based evidence. Prog Cardiovasc Nurs. $2005 ; 20(1): 17-23$..

60. Cahalin LP, Ferreira DC, Yamada S, Canavan PK. Review of the effects of resistance training in patients with chronic heart failure: potential effects upon the muscle hypothesis. Cardiopulm Phys Ther J. 2006;17(1):15-28.

61. Spruit MA, Eterman RM, Hellwig VA, Janssen PP, Wouters EF, Uszko-Lencer NH. Effects of moderate-to-high intensity resistance training in patients with chronic heart failure. Heart. 2009;95(17):1399-1408.

62. Clark AM, Hartling L, Vandermeer B, McAlister FA. Meta-analysis: secondary prevention programs for patients with coronary artery disease. Ann Intern Med. 2005;143(9):659-672.

63. Cornish AK, Broadbent S, Cheema BS. Interval training for patients with coronary artery disease: a systematic review. Eur J Appl Physiol. 2011;111(4):579-589.

64. Cortes OL, Villar JC, Devereaux PJ, DiCenso A. Early mobilisation for patients following acute myocardiac infarction: a systematic review and meta-analysis of experimental studies. Int J Nurs Stud. 2009;46(11):1496-1504.

65. Haykowsky M, Scott J, Esch B, et al. A meta-analysis of the effects of exercise training on left ventricular remodeling following myocardial infarction: start early and go longer for greatest exercise benefits on remodeling. Trials. 2011;12:92. 
66. Jolliffe JA, Rees K, Taylor RS, Thompson D, Oldridge N, Ebrahim S. Exercise-based rehabilitation for coronary heart disease. Cochrane Database Syst Rev. 2009;(1):CD001800.

67. Oliveira JL, Galvão CM, Rocha SM. Resistance exercises for health promotion in coronary patients: evidence of benefits and risks. Int $J$ Evid Based Healthc. 2008;6(4):431-439.

68. Valkeinen H, Aaltonen S, Kujala UM. Effects of exercise training on oxygen uptake in coronary heart disease: a systematic review and meta-analysis. Scand J Med Sci Sports. 2010;20(4):545-555.

69. Watson L, Ellis B, Leng GC. Exercise for intermittent claudication. Cochrane Database Syst Rev. 2008;(4):CD000990.

70. Wind J, Koelemay MJ. Exercise therapy and the additional effect of supervision on exercise therapy in patients with intermittent claudication. Systematic review of randomised controlled trials. Eur $J$ Vasc Endovasc Surg. 2007;34(1):1-9.

71. Kelley GA, Kelley KA, Tran ZV. Aerobic exercise and resting blood pressure: a meta-analytic review of randomized, controlled trials. Prev Cardiol. 2001;4(2):73-80.

72. Cornelissen VA, Fagard RH. Effects of endurance training on blood pressure, blood pressure-regulating mechanisms, and cardiovascular risk factors. Hypertension. 2005;46(4):667-675.

73. Dickinson HO, Mason JM, Nicolson DJ, et al. Lifestyle interventions to reduce raised blood pressure: a systematic review of randomized controlled trials. J Hypertens. 2006;24(2):215-233.

74. Whelton SP, Chin A, Xin X, He J. Effect of aerobic exercise on blood pressure: a meta-analysis of randomized, controlled trials. Ann Intern Med. 2002;136(7):493-503.

75. Witham MD, Avenell A. Interventions to achieve long-term weight loss in obese older people: a systematic review and meta-analysis. Age Ageing. 2010;39(2):176-184.

76. Shaw K, Gennat H, O'Rourke P, Del Mar C. Exercise for overweight or obesity. Cochrane Database Syst Rev. 2006;(4):CD003817.

77. Ottawa Panel. Evidence-based clinical practice guidelines for therapeutic exercises and manual therapy in the management of osteoarthritis. Phys Ther. 2005;85(9):907-971.

78. Brosseau L, Pelland L, Wells G, et al. Efficacy of aerobic exercises for osteoarthritis (part II): a meta-analysis. Phys Ther Rev. 2004;9(3): $125-145$.

79. Devos-Comby L, Cronan T, Roesch SC. Do exercise and selfmanagement interventions benefit patients with osteoarthritis of the knee? A metaanalytic review. J Rheumatol. 2006;33(4):744-756.

80. Lange AK, Vanwanseele B, Fiatarone Singh MA. Strength training for treatment of osteoarthritis of the knee: a systematic review. Arthritis Rheum. 2008;59(10):1488-1494.

81. Pelland L, Brosseau L, Wells G, et al. Efficacy of strengthening exercises for osteoarthritis (part I): a meta-analysis. Phys Ther Rev. 2004;9(2):77-108

82. de Kam D, Smulders E, Weerdesteyn V, Smits-Engelsman BC. Exercise interventions to reduce fall-related fractures and their risk factors in individuals with low bone density: a systematic review of randomized controlled trials. Osteoporos Int. 2009;20(12):2111-2125.

83. Li WC, Chen YC, Yang RS, Tsauo JY. Effects of exercise programmes on quality of life in osteoporotic and osteopenic postmenopausal women: a systematic review and meta-analysis. Clin Rehabil. 2009; 23(10):888-896

84. Boulé NG, Kenny GP, Haddad E, Wells GA, Sigal RJ. Meta-analysis of the effect of structured exercise training on cardiorespiratory fitness in type 2 diabetes mellitus. Diabetologia. 2003;46(8):1071-1081.
85. Chudyk A, Petrella RJ. Effects of exercise on cardiovascular risk factors in type 2 diabetes: a meta-analysis. Diabetes Care. 2011;34(5): $1228-1237$.

86. Irvine C, Taylor NF. Progressive resistance exercise improves glycaemic control in people with type 2 diabetes mellitus: a systematic review. Aust J Physiother. 2009;55(4):237-246.

87. Kelley GA, Kelley KS. Effects of aerobic exercise on lipids and lipoproteins in adults with type 2 diabetes: a meta-analysis of randomized-controlled trials. Public Health. 2007;121(9):643-655.

88. Snowling NJ, Hopkins WG. Effects of different modes of exercise training on glucose control and risk factors for complications in type 2 diabetic patients: a meta-analysis. Diabetes Care. 2006;29(11): 2518-2527.

89. Thomas DE, Elliott EJ, Naughton GA. Exercise for type 2 diabetes mellitus. Cochrane Database Syst Rev. 2009;(3):CD002968.

90. Umpierre D, Ribeiro PA, Kramer CK, et al. Physical activity advice only or structured exercise training and association with $\mathrm{HbA}_{1 \mathrm{C}}$ levels in type 2 diabetes: a systematic review and meta-analysis. JAMA. 2011;305(17):1790-1799.

91. O'Brien K, Nixon S, Tynan AM, Glazier RH. Aerobic exercise interventions for people living with HIV/AIDS: implications for practice, education, and research. Physiother Can. 2006;58(2):114-129.

92. Lawlor DA, Hopker SW. The effectiveness of exercise as an intervention in the management of depression: systematic review and meta-regression analysis of randomised controlled trials. $B M J$. 2001;322(7289):763-767.

93. Krogh J, Nordentoft M, Sterne JA, Lawlor DA. The effect of exercise in clinically depressed adults: systematic review and meta-analysis of randomized controlled trials. J Clin Psychiatry. 2011;72(4): $529-538$.

94. Herring MP, O'Connor PJ, Dishman RK. The effect of exercise training on anxiety symptoms among patients: a systematic review. Arch Intern Med. 2010;170(4):321-331.

95. Rethorst CD, Wipfli BM, Landers DM. The antidepressive effects of exercise: a meta-analysis of randomized trials. Sports Med. 2009;39(6):491-511.

96. Mead GE, Morley W, Campbell P, Greig CA, McMurdo M, Lawlor DA. Exercise for depression. Cochrane Database Syst Rev. 2008;(4):CD004366.

97. Centre for Evidence Based Medicine. Levels of Evidence. EBM Tools. Available at http://www.cebm.net/index.aspx?o=1025. Accessed February 29, 2012.

98. Marciniuk DD, Brooks D, Butcher S, et al. Optimizing pulmonary rehabilitation in COPD - practical issues: a Canadian Thoracic Society Clinical Practice Guideline. Can Respir J. 2010;17(4):159-168.

99. Ora J, Laveneziana P, Ofir D, Deesomchok A, Webb KA, O'Donnell DE. Combined effects of obesity and chronic obstructive pulmonary disease on dyspnea and exercise tolerance. Am J Respir Crit Care Med. 2009;180(10):964-971.

100. Roig M, Eng JJ, MacIntyre DL, et al. Falls in people with chronic obstructive pulmonary disease: an observational cohort study. Resp Med. 2011;105(3):461-469.

101. Pitta F, Troosters T, Probst VS, Langer D, Decramer M, Gosselink R. Are patients with COPD more active after pulmonary rehabilitation? Chest. 2008;134(2):273-280. 


\section{Appendix I \\ Search strategy for Medline Ovid}

Most of the numbered items are entered as keywords or phrases in the search box on the main page of Medline OvidSP. However, some line items at the end of each concept (eg, line 7) are commands to combine previous items in a particular fashion for the main concept. Asterisks indicate truncated terms in Medline. As well, abbreviations (ie, adj3) can be used to link words together.

Similar search terms were used in all databases. However, the preferred term could differ and the user needed to determine if the alternate term provided in a database was suitable. In addition, the manner of truncation, labeling for title or abstract, and combining of terms differed amongst databases.

\section{Concept: COPD}

1. lung diseases, obstructive/or exp pulmonary disease, chronic obstructive/or bronchitis, chronic/

2. exp Pulmonary Emphysema/

3. (obstruct* adj3 (pulmonary or lung* or airway* or airflow* or bronch* or respirat*)).ti,ab.

4. ((chronic airflow or chronic airway*) adj2 (disease* or disorder* or obstruct* or limitation*)).ti,ab.

5. (COPD or COAD or chronic bronchi* or emphysema* or hyperlucent lung*).ti,ab.

6. (chronic adj3 obstructive).ti,ab.

7. or/ $1-6$

\section{Concept: comorbidities}

8. bronchial diseases/or asthma/or bronchial hyperreactivity/ or bronchial spasm/or bronchiectasis/or exp bronchitis/ or bronchopneumonia/

9. (bronchial disease* or asthma* or bronchial asthma* or bronchial hyperreactivit* or bronchospasm*).ti,ab.

10. (bronchiectas* or bronchitis or bronchopneumonia* or bronchial pneumonia*).ti,ab.

11. exp Pneumonia/

12. (pneumonia* or pneumoniti* or pulmonary inflammation* or lung inflammation* or lobar pneumonia*). ti,ab.

13. Influenza, Human/

14. ((influenza adj3 human*) or human flu or influenza* or grippe).ti,ab.

15. respiratory insufficiency/

16. (respiratory insufficiency or respiratory failure or respiratory depression).ti,ab.
17. heart diseases/or arrhythmias, cardiac/or heart failure/ or myocardial ischemia/or myocardial infarction/or pulmonary heart disease/

18. (arrythmia or arrhythmia or cardiac arrhythmia* or cardiac dysrhythmia*).ti,ab.

19. (heart failure or cardiac failure or myocardial failure or heart decompensation or myocardial infarction).ti,ab.

20. (pulmonary heart disease* or cor pulmonale).ti,ab.

21. Hypertension/

22. (hypertension or high blood pressure).ti,ab.

23. Diabetes Mellitus/

24. (diabetes mellitus or diabetes).ti,ab.

25. Obesity/or Overweight/

26. (obesity or overweight).ti,ab.

27. Sleep Apnea, Obstructive/

28. (sleep apnea or obstructive sleep apnea or obstructive sleep apnea syndrome).ti,ab.

29. Depression/

30. (depression* or depressive symptoms or emotional depression*).ti,ab.

31. Anxiety/

32. (anxiet* or nervousness or anxious).ti,ab.

33. Anemia/

34. (anemia* or anaemia).ti,ab.

35. Osteoporosis/

36. (osteoporos\#s or senile osteoporos\#s or bone-loss*). ti,ab.

37. Osteoarthritis/

38. (osteoarthritis or osteoarthritides or osteoarthros\#s or degenerative arthritis).ti,ab.

39. osteopenia.mp.

40. exp aged/or frail elderly/

41. (aged or elderly or frail elderly or frail elder* or frail older adult*).ti,ab.

42. or $/ 8-41$

43. 7 or 42

\section{Concept: hospitalized patients}

44. exp Inpatients/

45. Hospitalization/

46. (inpatient* or in-patient* or hospitalized or hospitalised or hospitalization*).ti,ab.

47. or $/ 44-46$

48. 43 and 47

\section{Concept: exercise}

49. exp exercise/or exp walking/

50. exp Exercise Therapy/ 


\section{Physical Fitness/}

52. Exercise Movement Techniques/

53. (exercise* or physical exercise* or exercise therap* or walk* or ambulat* or physical fitness or physical activity).ti,ab.

54. (exercise* adj5 train*).mp.

55. (strength* adj5 train*).mp.

56. exp "Physical Therapy (Specialty)"।

57. (physiother* or physical therap*).mp.
58. Rehabilitation/
59. rehabilitat*.mp.
60. or/49-59
61. 48 and 60
62. animals/not humans/
63. 61 not 62
64. limit 63 to English

\section{Publish your work in this journal}

The International Journal of COPD is an international, peer-reviewed journal of therapeutics and pharmacology focusing on concise rapid reporting of clinical studies and reviews in COPD. Special focus is given to the pathophysiological processes underlying the disease, intervention programs, patient focused education, and self management protocols.
This journal is indexed on PubMed Central, MedLine and CAS. The manuscript management system is completely online and includes a very quick and fair peer-review system, which is all easy to use. Visit http://www.dovepress.com/testimonials.php to read real quotes from published authors. 